\title{
Incidencia del gasto público en $I+D+i$ energético sobre la corrección medioambiental en España*
}

\section{JOSÉ Ma CANTOS}

Departamento de Economía Política y Hacienda Pública, E.E. y P.E., UNIVERSIDAD DE CASTILLA LA MANCHA, ESPAÑA.E-mail: JoseMaria.Cantos@uclm.es

\section{DANIEL BALSALOBRE LORENTE}

Departamento de Economía Política y Hacienda Pública, E.E. y P.E., UNIVERSIDAD DE CASTILLA LA MANCHA, ESPAÑA. E-mail: Daniel.Balsalobre@uclm.es

\begin{abstract}
RESUMEN
Durante los últimos años la política energética española ha estado muy sesgada hacia la sustitución de fuentes energéticas tradicionales, muy contaminantes, por otras renovables, sin índice de carbonización. La gran cantidad de recursos públicos que se han destinado a financiar la producción de estas energías contrasta con la creciente escasez general de recursos y con la reducida aportación pública a la $\mathrm{I}+\mathrm{D}+\mathrm{i}$ en el sector energético español. Utilizando el modelo de Curva de Kuznets Ambiental, hemos obtenido evidencia sobre la existencia de una relación inversa entre el gasto público en políticas de $\mathrm{I}+\mathrm{D}+\mathrm{i}$ energéticas y las emisiones de gases contaminantes.

Palabras clave: Energías renovables, eficiencia energética, gasto público en I+D+i energético.
\end{abstract}

\section{Impact of Public R\&D in Energy on Environmental Correction in Spain}

\begin{abstract}
During the last years the Spanish energy policy has been heavily biased towards replacing traditional energy sources, highly polluting, other renewable, without charring rate. The large amount of public resources being allocated to finance the production of these energy contrasts with the growing general shortage of resources and reduced public input to R\&D in the spanish energy sector. Using, for empirical analysis, the model of Environmental Kuznets Curve, we obtained evidence for existence of an inverse relationship between public spending policies R\&D energy and greenhouse gas emissions.
\end{abstract}

Keywords: Renewable Energy, Energy Efficiency, Public Expenditure on Energy R\&D.

Clasificación JEL: P28, P48, Q48

\footnotetext{
* Los autores quieren expresar su agradecimiento a dos evaluadores anónimos que han contribuido significativamente a mejorar el contenido y la presentación de este trabajo.
}

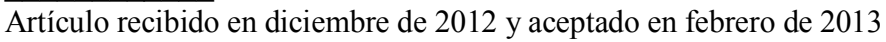

Artículo disponible en versión electrónica en la página www.revista-eea.net, ref. ə-31108 


\section{SISTEMA ENERGÉTICO E INTERVENCIÓN PÚBLICA}

Desde las primeras décadas del siglo XX, en muchos países se consideró que el sistema energético constituía un sector estratégico de las economías nacionales por diversas razones. Primero, porque su carácter horizontal para el resto de sectores impone la necesidad de garantizar el abastecimiento de las principales fuentes, desigualmente repartidas por el mundo, con el fin de evitar un colapso derivado de comportamientos estratégicos hostiles de los países productores (seguridad de abastecimiento). En segundo lugar, los requerimientos tecnológicos de la producción y distribución de muchas de las fuentes energéticas, incluso dentro del propio país, constituyen un elemento distorsionador para un mercado de libre competencia, generando posiciones dominantes capaces de alterar el precio que se obtendría en condiciones competitivas (fallos de mercado).

Por lo que se refiere a este segundo motivo, es cierto que en el plano teórico cabe cuestionar que la competencia perfecta deba someterse siempre al requisito de la concurrencia de muchos oferentes y muchos demandantes, para acercarse más a la idea de libre competencia esbozada ya hace muchas décadas por algunos representantes de la Escuela Austríaca, consistente en la necesidad de relajar la exigencia formal de una multitud de concurrentes en ambos lados del mercado, por otra más centrada en el grado de concurrencia posible que permita las condiciones técnicas objetivas de cada mercado ${ }^{1}$. En todo caso, lo cierto es que las imperfecciones que se dan en la práctica totalidad del resto de mercados impedirían alcanzar la efíciencia en el mercado energético para la inmensa mayoría de economías actuales. El recurso a la teoría de los fallos del mercado parece inevitable en un mundo como el actual, y lo que sería exigible, en todo caso, es que la intervención pública fuera eficiente.

La sostenibilidad del crecimiento económico comienza a ocupar la agenda de ciertos organismos internacionales, sobre todo, a partir de la creación del Club de Roma (1968), con su publicación de renombre mundial en 1972, a pesar de que no ha podido contrastarse el catastrofismo que se desprendía de sus conclusiones $^{2}$. A lo largo de las últimas décadas, el movimiento en torno a la sostenibilidad del crecimiento ha ido permeabilizando cada vez más los objetivos de las políticas públicas, hasta el punto de que ha surgido con fuerza una tercera razón a tener en cuenta a la hora de considerar al sector energético como

\footnotetext{
${ }^{1}$ En relación con la concepción austríaca del término "competencia perfecta" acuñado desde los clásicos, véase Rothbard, M. N. (1970).

${ }^{2}$ Véase "Los límites del crecimiento", el primer informe de trascendencia encargado al MIT en 1972. Otros acontecimientos pioneros fueron la celebración de la primera "Cumbre de la tierra" (1972), en Estocolmo, la adopción de la "Carta Mundial de la ONU para la Naturaleza" (1982), la creación en el seno de la ONU de la Comisión Mundial sobre Medio Ambiente y Desarrollo (1983), o el denominado Informe Brundtland (“Our common future") en 1987.
} 
un sector estratégico: la necesidad de lograr una producción y un consumo de energía sostenibles, por cuanto que en ningún otro sector como en éste se personaliza de manera tan nítida el impacto medioambiental negativo de los procesos económicos.

Por tanto, la acción pública en el campo energético cuenta con tres poderosas razones que la respaldan: la estratégica, la necesidad de lograr un mercado eficiente y la de controlar el impacto medioambiental, si bien, una buena parte de la última bien podría subsumirse en la segunda a modo de restricción, toda vez que implica la internalización de los costes ambientales en los procesos de producción, distribución y consumo. Desde el punto de vista formal, el ejercicio de optimización podría consistir en determinar la cantidad mínima de energía a producir (y consumir) dadas unas necesidades de crecimiento económico, sujeto a una estructura de costes determinada basada en un sistema de precios socialmente eficientes. Sin embargo, la sencillez que se desprende de la exposición del problema se encuentra tan alejada de la realidad que resultaría poco práctico, por ineficaz, aproximarse al problema de esta forma global, además de los recelos que suscita el empleo de un enfoque del tipo "dictador benevolente". Es preciso, por tanto, aproximarse a esta cuestión con un enfoque parcial.

Cuando nos enfrentamos al problema económico de analizar una medida de política energética, se precisa ir más allá del puro resultado en términos de eficacia, una vez fijado y cuantificado el objetivo, y una vez fijados y acotados los indicadores de costes. En nuestro caso, el objeto de interés se centra en el impacto que ciertas medidas públicas (las de $\mathrm{I}+\mathrm{D}+\mathrm{i}$ ) adoptadas en España han tenido sobre la reducción de emisiones contaminantes asociadas a los procesos de producción y consumo de energía, dentro de un contexto de adaptación a las exigencias de la Unión Europea ${ }^{3}$. Para conseguir el objetivo final de sostenibilidad pueden establecerse, grosso modo, dos objetivos intermedios: reducir la producción y/o consumo de las fuentes energéticas más contaminantes en favor de otras fuentes más respetuosas con el medio ambiente; o bien, conseguir mejorar la eficiencia energética general del sistema económico.

En cuanto al primero de estos objetivos, la sustitución de fuentes contaminantes (básicamente de origen fósil) puede realizarse mediante la promoción de fuentes de energía nuclear o de otras renovables, cuyas emisiones contaminantes son significativamente menores (IDAE, 2008). Dos problemas surgen cuando se adopta esta estrategia: por un lado, el mas o menos presente rechazo social que ocasiona la producción nuclear, y por otro, el diferencial penalizador de costes que representan el resto de fuentes renovables por unidad energética

\footnotetext{
${ }^{3}$ Entre las normas dictadas por la UE en materia de eficiencia energética, se encuentran la Directiva 2002/91/CE, relativa a la eficiencia energética de los edificios, y la Directiva 2006/32/CE, sobre eficiencia del uso final de la energía y los servicios energéticos.
} 
producida, lo que impulsa un aumento considerable en el coste tarifario ${ }^{4}$. Por lo que se refiere a la energía nuclear, el alto coste de planta se compensa con los bajos costes unitarios de producción, algo que está fuera de toda duda si se computan exclusivamente los costes financieros, pero que suscita no poca controversia cuando se contemplan, siquiera en el plano formal, los costes asociados al riesgo de catástrofe radiactiva, teniendo en cuenta que este tipo de cálculos son difíciles de realizar debido a las extraordinarias secuelas que provocarían, además, por tiempo indefinido. Por su parte, el uso de energías renovables en la actualidad tiene como contrapunto un coste unitario significativamente superior, lo que conlleva, en el mejor de los casos, que la sustitución energética eficiente no tendrá lugar sino en el largo plazo ${ }^{5}$. La intervención pública se enfrenta, por tanto, a la disyuntiva de interiorizar costes allí donde es posible y dejar que sea el mercado el que elija el mix energético, o sustituir la voluntad del mercado promocionando un determinado tipo de fuentes renovables en detrimento de otras, a sabiendas de que este último comportamiento va más allá de la licencia obtenida con cargo a la teoría de los fallos del mercado.

El segundo de los objetivos intermedios que puede plantearse la intervención administrativa es mejorar la eficiencia energética general del sistema económico, esto es, se trata de conseguir reducir la ratio de energía consumida por unidad de PIB, medido por el índice de intensidad energética, lo que automáticamente redundaría en una reducción de las emisiones contaminantes, ceteris paribus. Esta estrategia incide en el lado de la demanda, modulando la demanda final de productores y del sector residencial. En cuanto a la demanda de los productores, el objetivo a alcanzar es la mejora tecnológica aplicada a los procesos de producción y de distribución de energía, así como a la fabricación y/o explotación de herramientas y máquinas utilizadas en otros procesos productivos, o destinadas al uso particular. En todo caso, lo que subyace en cualquier estrategia de mejora de la eficiencia energética es el papel clave que representa el gasto en $\mathrm{I}+\mathrm{D}+\mathrm{i}$, tanto público como privado (Mansfield y Switzer, 1994; Fer-

\footnotetext{
${ }^{4}$ Según los datos que viene manejando la patronal UNESA, tan solo el 45,6\% de la factura eléctrica corresponde al consumo de electricidad, mientras que el resto debe cubrir las primas a las energías renovables (22,5\%), el IVA y el Impuesto sobre la Electricidad (18,7\%), anualidades abonadas a consecuencia del déficit tarifario acumulado de años anteriores $(6,1 \%)$, compensaciones extra-peninsulares (3\%), y conceptos varios, incluida la moratoria nuclear, Elcogas, los planes de calidad y de ahorro y eficiencia energética (4,1\%). A su vez, el coste de las primas es casi el equivalente al coste total de generación $(24,8 \%)$, incluido dentro del $45,6 \%$ ya citado.

${ }^{5}$ Por lo que se refiere al diferente coste financiero de producción por fuentes energéticas (en euros por $\mathrm{Mw} / \mathrm{h}$ ), los datos proporcionados por Enerclub para 2008 indican un coste de 430 para la solar fotovoltaica, 84 para la eólica, 60 para el gas, 52 para el carbón, 45 para la hidráulica y 36 para la nuclear. Dicho de otro modo, el coste del kw/h de la solar fotovoltaica representa casi 12 veces el de la nuclear, 9,5 veces el de la hidráulica y 8,3 veces el del carbón.
} 
nández, 2007; Hamilton y Turton, 2002; Fisher-Vanden 2004; Yuxiang y Chen, 2010; Ruiz, 2010).

Por lo que se refiere al conjunto de demandantes finales de energía, puede decirse que hay dos tipos de medidas que inciden de manera especial sobre la eficiencia. Por un lado, la incorporación de los costes externos a la tarifa regulada, así como una adecuada estructura de la misma, de manera que evidencie de forma clara ante los agentes económicos los incentivos para perseguir un comportamiento eficiente. Por otro lado, las campañas de concienciación ciudadana sobre consumo responsable (y de apoyo público, en su caso), también inciden positivamente en la mejora de la eficiencia energética. En todo caso, en las medidas de promoción de la eficiencia energética es aconsejable no perder de vista que el uso de campañas de concienciación basadas en la sustitución subvencionada de bienes de producción o de consumo final, solo son eficientes (incluso eficaces) cuando previamente se ha establecido una escala de precios regulados que proporcione incentivos en la misma dirección que las proporcionadas por esas medidas de concienciación y apoyo directo.

En relación con las medidas que contribuyen a que la escala de precios finales proporcione incentivos a la eficiencia, parece existir consenso en la literatura económica sobre la idea de una necesaria adecuación del precio final al coste real del servicio, algo que técnicamente sería muy sencillo si los propios costes financieros en la fase de producción, transporte, distribución y consumo de la energía, reflejaran en su integridad los costes totales. Sabemos que esto no suele ocurrir, puesto que existen efectos externos negativos (básicamente derivados de la contaminación ambiental) no computados, y una tarifa de precios finales socialmente eficiente debería incluir tal impacto negativo si se pretende que los incentivos que proporcione al demandante final de energía vayan en la misma dirección que el resto de incentivos proporcionados por otras medidas complementarias. Ahora bien, mientras que técnicamente es posible localizar y determinar, en la mayoría de las ocasiones, los costes no financieros asociados a los procesos de producción y transporte de la energía, resulta más difícil localizar y cuantificar dichos costes asociados al consumo final cuando la energía se utiliza, a su vez, como input en la producción de bienes y servicios. Para estos casos y para el resto de casos de uso final de la energía (uso residencial), existe una herramienta que permite incorporar los efectos externos negativos a la tarifa de precios finales: los impuestos especiales sobre el consumo de energía. Si la tarifa final recoge adecuadamente los costes financieros y no financieros, entonces generará incentivos para mejorar la eficiencia energética. En caso contrario, el efecto beneficioso sobre la eficiencia que pudieran tener otras medidas de política energética, quedarán contrarrestados y el sistema energético, en general, y los impuestos especiales, en particular, serán ineficientes. 
Por eso, una regulación energética adecuada requiere de una herramienta útil para lograr la eficiencia (Linares, 2002), generando incentivos en los agentes. Dentro de estos incentivos cabe resaltar los del cambio tecnológico, que permite mejoras permanentes en la eficiencia.

Otro tipo de instrumentos al servicio de la mejora en la eficiencia son los denominados instrumentos económicos de cantidad, donde el regulador busca reducir la cantidad consumida de energía. Se trata de medidas que imponen objetivos a las empresas distribuidoras o comercializadoras de energía, con ciertos grados de libertad para elegir la forma de alcanzar el objetivo, permitiendo a las empresas comerciar con las ganancias de eficiencia obtenidas, previamente su acreditación a través de una especie de certificado, dando lugar a un mercado secundario ${ }^{6}$.

Un tercer bloque de medidas regulatorias pasa por el establecimiento de estándares mínimos obligatorios, referidos a un nivel determinado de eficiencia mínima en equipos, edificios o procesos industriales ${ }^{7}$.

Finalmente, se encuentran las medidas destinadas a mejorar la información y la sensibilización de los consumidores por la eficiencia energética, por medio de campañas de información, la introducción de conceptos de eficiencia energética en los materiales educativos, la inclusión del etiquetado energético en equipamientos, la inclusión en las facturas de información sobre consumos históricos, la financiación de equipos de bajo consumo, o el fomento de auditorías energéticas.

\section{PRINCIPALES ASPECTOS DE LA POLÍTICA ENERGÉTICA RECIENTE EN ESPAÑA}

La Agencia Internacional de la Energía (AIE) viene identificando a lo largo de sus informes que existen una serie de factores vinculados a la emisión de gases de efecto invernadero (GEI) que provocan un cambio climático, y ha realizado proyecciones augurando importantes efectos adversos a largo plazo ${ }^{8}$. La

\footnotetext{
${ }^{6}$ Aunque este tipo de medidas estaban previstas en la Directiva 2006/32/CE, la Comisión Europea se muestra muy cautelosa al respecto y todavía se está analizando si realmente se trata de una herramienta adecuada para mejorar la eficiencia energética. En España no se han puesto en marcha.

${ }^{7}$ En Gillingham, Newell y Palmer (2009) se concluye que los estándares energéticos aplicados a electrodomésticos constituyen una de las medidas más eficientes para la reducción del consumo. En el transporte, merece destacar el Reglamento europeo aprobado en Diciembre de 2008, donde se establece un límite de emisiones de 120 gramos de $\mathrm{CO}_{2}$ por $\mathrm{km}$, en el año 2012, para toda la industria del automóvil, fijándose una sanción de $20 €$ por gramo y kilómetro que supere el límite de 130 gramos. Para 2013, la sanción asciende a 35 euros, a 60 euros en 2014 y a 95 euros en 2015.

${ }^{8}$ Basándose en el análisis del Panel Intergubernamental para el Cambio Climático (IPPC, siglas en inglés), la AIE plantea como escenario energético alternativo (BLUE) aquel que contempla
} 
propia política energética de la Unión Europea viene estableciendo una serie de directrices en orden a la reducción de las emisiones contaminantes, aportando también una serie de recomendaciones encaminadas a lograr este amplio objetivo y que inciden, tanto en la sustitución progresiva de fuentes energéticas contaminantes, como en la adopción de medidas que mejoren la eficiencia energética ${ }^{9}$. Sin embargo, la experiencia obtenida a partir de la intervención regulatoria llevada a cabo en España en los últimos 15 años, ha estado orientada, mayoritariamente, a la promoción de las energías renovables por encima de cualesquiera otras.

Figura 1.1

Gasto público español I+D+i energético $\left(10^{6} €\right.$ de 2010$)$

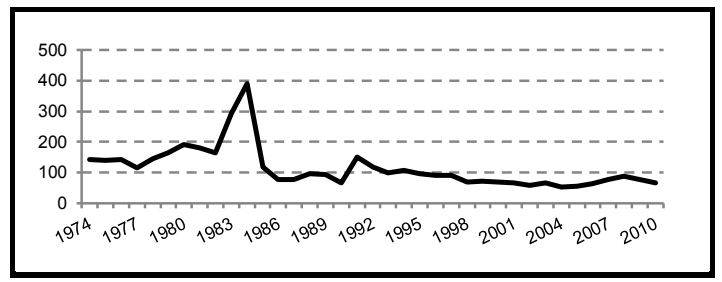

Fuente: OECD (2012).

Figura 1.2

Gasto público en I+D+i energético por países $\left(10^{6} €\right.$ de 2010$)$

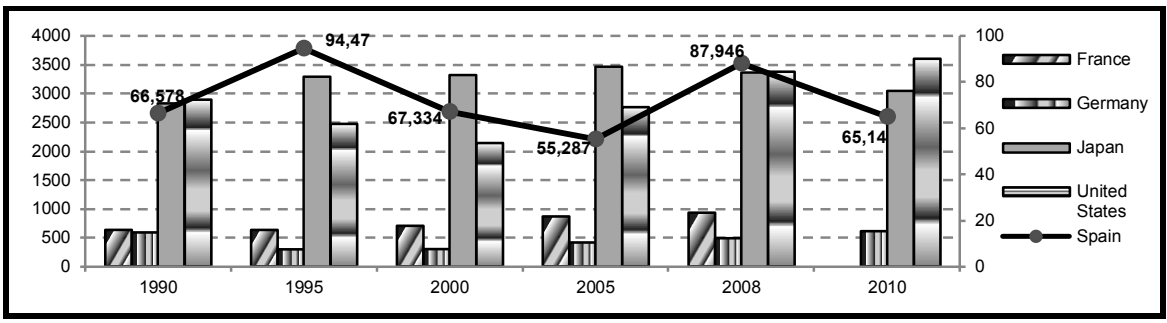

Nota: Los datos para España en eje derecho; los datos para resto de países (Francia, Alemania, Japón, EE.UU.) en eje izquierdo (millones de euros). Datos en millones de euros.

Fuente: OECD (2012).

una reducción de las emisiones de Gases de Efecto Invernadero (GEI) del 50\% para limitar el calentamiento global hasta 2-4 grados centígrados a finales de siglo. Así, según el último informe sobre Prospectiva de Tecnologías Energéticas 2008-2050 de la AIE, dentro del escenario BLUE, se concluye que una mejora en la eficiencia energética podría contribuir a la reducción global de emisiones en casi un $50 \%$.

${ }^{9}$ La Directiva 2006/32/CE estableció los aspectos básicos para regular los certificados de emisiones y fija un objetivo mínimo de ahorro energético del $9 \%$ en 2016, mientras que la Directiva 2009/28/CEE proponía objetivos a largo plazo para los Estados miembros en materia de producción renovable (en el caso de España, debería alcanzarse el 20\%). 
Como se muestra en la Figura 1.1, el esfuerzo en el gasto público de $\mathrm{I}+\mathrm{D}+\mathrm{i}$ energético, en términos reales, no ha experimentado cambios importantes a lo largo del período, salvo durante algunos años de la $2^{\mathrm{a}}$ crisis del petróleo (19831984), y en el período 1991-1992, manteniéndose en niveles muy reducidos en relación a otros países desarrollados (véase Figura 1.2). Además, si observamos la descomposición de dicho gasto público durante la última década, se aprecia cómo el esfuerzo ha sido más intenso en energías renovables y en la nuclear, mientras que el apoyo a las medidas de mejora de la eficiencia energética ha sido menor (Figura 2).

Figura 2

Gasto público en $1+D+\mid$ energético en España por objetivo de la investigación (1990-2008)

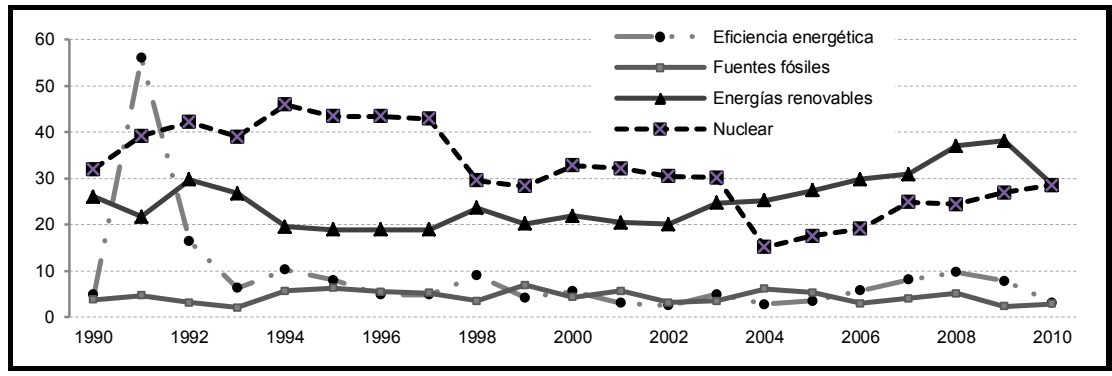

Nota: En millones de euros de 2010.

Fuente: OECD (2012).

En este sentido, resulta evidente la preferencia de la política energética española por el objetivo intermedio de la sustitución de fuentes, lo que ha dado lugar a una peculiar composición de los costes incluidos en la tarifa eléctrica (véase la nota al pie $\mathrm{n}^{\mathrm{o}} 4$ ), con el agravante de que no se ha llegado a trasladar a la propia tarifa todo el coste que representan las primas al régimen especial de generación, sino tan solo la anualidad financiera correspondiente a la enorme deuda acumulada que puede alcanzar la cifra de 29.000 millones de euros al finalizar $2012^{10}$. En consecuencia, el impacto de la política energética hace que el sistema en su conjunto esté teniendo unos costes que más que duplican los de generación y que, progresivamente, se están haciendo cada vez más visibles para los demandantes finales de energía eléctrica. Además, esta tendencia observada

${ }^{10}$ El Real Decreto 14/2010 prevé que el déficit de tarifa debe anularse para 2013, lo que implica que el coste total de la producción y distribución de la electricidad deberá trasladarse necesariamente a la tarifa eléctrica. Sin embargo, el déficit anual acumulado a fecha de agosto de 2012 ya alcanzaba los 3.611 millones de euros, superando ampliamente los 1.500 millones autorizados para el conjunto del año. 
en los últimos años se está viendo reforzada por la fuerte y persistente subida de los precios del crudo, acompañada de una gran volatilidad que obedece, en una parte importante, al repunte que ha experimentado la inestabilidad política en las regiones productoras. Aunque la alta volatilidad en los mercados del crudo no es nueva, puesto que viene arrastrándose desde la década de los pasados años setenta, la tendencia sostenida del precio al alza está potenciando una reorientación hacia el autoabastecimiento energético por parte de muchos países occidentales que no poseen recursos fósiles, reforzando de este modo las medidas motivadas por razones ambientales.

El actual panorama energético en España se caracteriza por una elevada dependencia de las importaciones de fuentes energéticas fósiles, superior al $80 \%$ del total, así como por unas necesidades de corrección de emisiones contaminantes que todavía son importantes a pesar del estancamiento del PIB real durante 4 años. A estas circunstancias se suma el hecho de que el precio final de la electricidad en España se encuentra por encima de la media europea, a lo que ha contribuido el sobrecoste derivado de las medidas de sustitución de fuentes impulsadas a lo largo de la última década (véase nota 4). Todo ello está elevando a la categoría de imprescindible la apuesta decidida por el otro objetivo intermedio de la política energética, hasta ahora casi abandonado: la consecución de mejoras de eficiencia en el sistema en su conjunto.

Esta preferencia por las medidas regulatorias centradas en la sustitución de fuentes, ha supuesto un considerable desgaste para el sector de las energías renovables al coincidir en unos pocos años dos circunstancias con implicaciones opuestas: la gran intensidad con que se han venido implantando las fuentes renovables, y la llegada de la profunda y pertinaz crisis económica. El choque entre la elevada cuantía que el Estado debe aportar en concepto de prima a la producción del régimen especial, y la creciente contención de los ingresos públicos, ha dado lugar a retoques a la baja en las primas a percibir, generando un clima de incertidumbre sobre el sector de las renovables que puede complicar más el cumplimiento de los compromisos medioambientales por parte de España. Probablemente, las duras circunstancias actuales constituyan algo más que una excusa para cambiar la estrategia energética, ponderando más la eficiencia que la sustitución de fuentes.

Creemos necesario que las autoridades energéticas lleven a cabo un estudio de coste beneficio para determinar la estrategia a seguir con el fin de alcanzar los objetivos energéticos finales, y evitar que sean los acontecimientos los que se pongan delante de la planificación para impulsar un cambio de estrategia más centrado en la mejora de la eficiencia energética. Fijarse como objetivo intermedio una mejora de la eficiencia implica actuaciones sobre empresas y sobre particulares, teniendo en cuenta que el ahorro esperado por la aplicación de estas medidas contribuye, no solo a reducir el nivel de emisiones de GEI a la 
atmósfera, sino que produce también desbordamientos (spillovers) positivos sobre la eficiencia en todo el sistema económico (Enerclub, 2009). Dentro de la estrategia a favor de la eficiencia, las medidas de oferta pasan necesariamente por aplicar un mayor esfuerzo en $\mathrm{I}+\mathrm{D}+\mathrm{i}$, aspecto que, en el terreno de la investigación económica, ha llenado durante décadas muchas páginas cuyas conclusiones mayoritariamente refrendan las tesis del impacto significativamente positivo de la innovación tecnológica sobre la eficiencia de cualquier proceso productivo. Si el gasto privado en $\mathrm{I}+\mathrm{D}+\mathrm{i}$ se estima como positivo en este terreno, entonces el apoyo público a estas iniciativas quedaría justificado (Porter y Van Der Linde, 1995).

El papel reservado a la intervención pública también pasa por incentivar el ahorro energético a través de diversos instrumentos regulatorios dirigidos tanto a productores como a demandantes finales de energía (empresas y particulares): formación de consumidores, activación del mercado del ahorro y la eficiencia energética, e inducción de acciones encaminadas en esta dirección. Entre la batería de instrumentos regulatorios se encuentran los instrumentos económicos de precio y cantidad, las medidas de "mandato y control", las medidas destinadas a mejorar la información y sensibilización de los consumidores, y la financiación de actividades de $\mathrm{I}+\mathrm{D}+\mathrm{i}$, ya sea de iniciativa pública o privada.

Solo desde fechas recientes se viene aplicando en España una estrategia de mejora de la eficiencia energética instrumentada por medio de los Planes de Acción para el Ahorro y la Eficiencia Energética: Plan 2005-2007, Plan 20082012 y el reciente Plan 2011-2020. Todos estos planes se basan en la financiación conjunta entre el sector público (Administración Central y Comunidades Autónomas) y el sector privado, aunque el esfuerzo público apenas ha representado $1.500 \mathrm{M} / €$ (millones de euros) durante el periodo 2005-2010, lo que supone una aportación anual de $250 \mathrm{M} / €$, cifra poco significativa si se compara con los $7.942 \mathrm{M} / €$ que costaron al sector público las renovables solo en 2010, o $\operatorname{los} 25.000 \mathrm{M} / €$ a que asciende el déficit de tarifa acumulado hasta $2012^{11}$.

E1 Plan de Acción 2008-2012 contemplaba un escenario que permitía alcanzar el objetivo fijado por la UE para España (mejora de eficiencia energética en un $20 \%$ para 2020), lo que requería que la tasa de crecimiento interanual del consumo en energía primaria debía reducirse hasta el 1,07\%. Sin embargo, la profundidad y duración de la actual crisis económica pronto ha dejado obsoletos los cálculos e, incluso, la estrategia general para el sector eléctrico. El Consejo de Ministros (29/7/2011) aprobó el Plan de Ahorro y Eficiencia Energética 2011-2020, de acuerdo con la Directiva 2006/32/CE, en lo que constituye el $2^{\circ}$ Plan de Acción Nacional. Este Plan incorpora un análisis coste-beneficio que

${ }^{11}$ Según declara la Vicepresidenta del Gobierno de España en rueda de prensa con motivo del Consejo de Ministros de 11 de enero de 2013. 
arroja unos beneficios económicos totales de $78.687 \mathrm{M} / €(8.330 \mathrm{M} / €$ derivados de las emisiones evitadas de $\mathrm{CO}_{2}$ y $70.357 \mathrm{M} / €$ por ahorro de energía primaria) para el conjunto del período, de los que el 53\% corresponde a la industria, el $22 \%$ a los procesos de transformación de la energía y el 19\% al transporte, con apenas impacto sobre la agricultura, los servicios públicos y el sector de edificación y equipamiento. De los cerca de $46.000 \mathrm{M} / €$ en que se valoran las necesidades de inversión, la aportación pública se estima en el 10,8\%, representando $552 \mathrm{M} / €$ por año, cuantía considerablemente superior a la asignada en la etapa anterior $(250 \mathrm{M} / €)$, a pesar del escenario de mayor austeridad presupuestaria que se vislumbra para las administraciones públicas durante los próximos años ${ }^{12}$.

Parece adecuado actuar sobre la demanda final de energía de las empresas con medidas como las descritas, pues no en vano absorben más del $33 \%$ de la energía consumida. Los últimos informes disponibles apuntan a comportamientos ineficientes en el sector de las PYMEs, donde poco más de la mitad lleva a cabo labores de mantenimiento de sus instalaciones, menos del $10 \%$ ha realizado auditorías energéticas y solamente el $27 \%$ ha implantado alguna medida de ahorro. Y esto sin olvidar el ahorro potencial de los hogares españoles, puesto que son responsables del $30 \%$ del consumo ${ }^{13}$; en este sentido, estimaciones realizadas ya en 2002 por UNESA, situaban el consumo energético en un $30 \%$ superior al consumo de un hogar medio en Europa. Finalmente, la parte más importante del consumo energético final corresponde al sector del transporte, con un $37 \%$, sector que es el principal responsable del incremento experimentado por el indicador de intensidad energética del sistema eléctrico en su conjunto (IDAE, 2011).

A partir de estos datos, parece existir un importante recorrido para las políticas de fomento del ahorro energético y de mejora de la eficiencia, teniendo en cuenta que esta estrategia resulta complementaria a la de sustitución de fuentes para conseguir alcanzar los objetivos finales, tanto en lo que se refiere a la reducción de emisiones de gases contaminantes, como a la reducción de la dependencia exterior. Se trataría de lograr que el índice de intensidad energética flexione a la baja a medio y largo plazo, con independencia de los vaivenes que provoca el ciclo económico, rompiendo una tendencia que hasta 2005 parecía no tener fin, sobre todo cuando para el cálculo del índice de intensidad energética se utiliza el indicador de energía final. Esto es lo que ilustra la Figura 3.1, donde la intensidad energética se calcula como el cociente entre el consumo

\footnotetext{
${ }^{12}$ Véase el Plan de Ahorro y Eficiencia Energética 2011-2020 (IDAE).

${ }^{13}$ Del 30\% del consumo energético realizado por las familias españolas, la mitad corresponde al uso del coche y el resto a los usos domésticos; dentro de éstos últimos, destacan los gastos destinados a calefacción y agua caliente sanitaria (ACS), aunque el consumo en climatización está aumentando muy rápidamente.
} 
energético y el PIB. En la serie que comienza en 1990, la intensidad energética ha seguido una senda ascendente, a lo que ha contribuido, entre otros factores, un significativo aumento en el nivel de equipamiento de los hogares españoles, aun cuando a partir de 2007 el cambio de ciclo económico parece haber afectado notablemente a la demanda de este tipo de bienes de consumo duradero ${ }^{14}$.

Figura 3.1

Intensidad energética: consumo de energía final por unidad de PIB (tep/millón €)

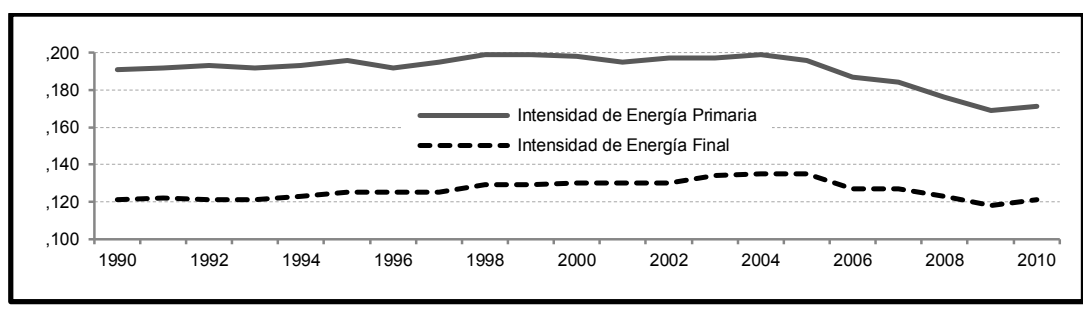

Nota: Metodología AIE (2012). PIB en miles de millones de euros constantes 2000

Fuente: Secretaría General de Energía (SGE).

Figura 3.2

Evolución de las intensidades energéticas sectoriales en España

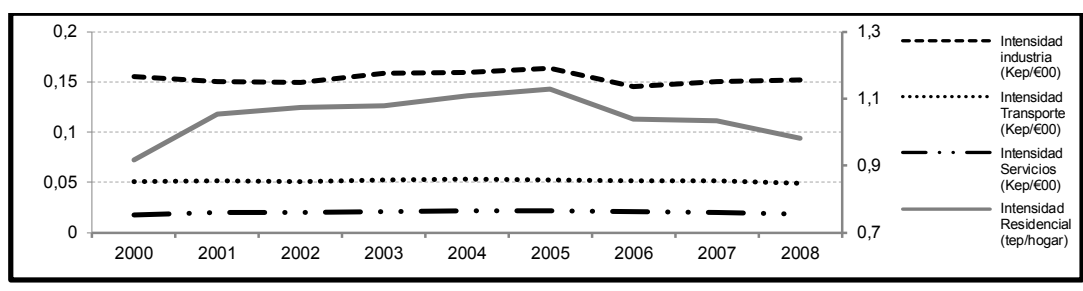

Nota: Metodología IDAE (2010). Eje derecho: intensidad residencial (toneladas equivalentes de petróleo/hogar). Eje izquierdo, kilotoneladas equivalentes petróleo/PIB2000).

Fuente: Secretaría General de Energía (SGE).

La evolución de la intensidad energética final en España en los años previos a la crisis, constata un alejamiento respecto a media de la UE (IDAE, 2011),

\footnotetext{
${ }^{14}$ La estadística de equipamiento de los hogares de Eurostat, así como otras fuentes de muestreo, como las encuestas que viene realizando la Cámara de Comercio de Madrid, ilustran una pujante evolución positiva en el nivel de equipamiento de electrodomésticos en los hogares. Con todo, por citar una fuente no oficial, resulta también interesante el estudio de seguimiento periódico sobre eficiencia energética que afecta solamente a los hogares españoles, y que lleva a cabo Unión Fenosa, donde se pone de manifiesto una mejora sostenida del índice del 8,66\% entre 2004 y 2011 , equivalente a un ahorro energético de $222 \mathrm{M} / €$ año, estimando un valor económico para el ahorro potencial de 2011 de $1.407 \mathrm{M} / €$.
} 
aunque el índice español comienza una senda convergente a partir de 2006. Según muestra la Figura 3.2, los sectores que apenas han experimentado cambios en su índice de intensidad energética han sido el sector del transporte y el de servicios; por otro lado, el sector industrial presenta una corrección a la baja a partir de 2006, al igual que ocurre con el sector residencial (eje derecho).

De acuerdo con estas cifras, la reciente aprobación del Plan de Acción de Ahorro y Eficiencia Energética 2011-2020, pretende incidir prioritariamente en el ahorro del sector industrial y en el del transporte, aunque las previsiones para este último son modestas (con una aportación del 19\% a la mejora total esperada), probablemente basadas en la dificultad que entraña una ganancia significativa de eficiencia en el corto y medio plazo. Con todo, de cumplirse las previsiones del Plan, implicaría un cambio en la buena dirección de la política energética, generando desbordamientos positivos en términos de una contribución al PIB nacional del 3,9\% en el período y la creación de 300.000 puestos de trabajo.

\section{EL PAPEL DEL I+D+i ENERGÉTICO Y LA SOSTENIBILIDAD MEDIOAMBIENTAL}

Como ya advertimos al principio, el sector de la energía suele desenvolverse en mercados muy alejados del concepto al uso de competencia perfecta, lo que de una manera $u$ otra afecta negativamente a las decisiones sobre inversión en $\mathrm{I}+\mathrm{D}+\mathrm{i}$. En este caso, a las incertidumbres propias de cualquier mercado, se suma la incertidumbre adicional derivada de un mercado tan regulado como el eléctrico, lejos, por tanto, de lo que pudiera pensarse a primera vista, como viene ocurriendo en España con la cambiante regulación en los subsectores de las renovables y en el energético nuclear. Entre los factores que alimentan la incertidumbre en el sector energético y que dificultan las inversiones en intangibles como la $\mathrm{I}+\mathrm{D}+\mathrm{i}$, tendríamos unos precios energéticos artificialmente bajos (al no incorporar todos los costes de suministro), los continuos cambios normativos que afectan a la rentabilidad de la inversión en intangibles, la escasa información sobre avances técnicos que genera dificultades para dar respuestas a la demanda, las propias imperfecciones en los mercados de capitales, o la falta de cultura científico-técnica y de innovación en España, por citar algunos.

El papel de la $\mathrm{I}+\mathrm{D}+\mathrm{i}$ en los procesos energéticos ha adquirido especial relevancia en la literatura económica durante los últimos años. Algunas de las aportaciones más interesantes se han preocupado por identificar los principales condicionantes de la variación de la intensidad energética (Mendiluce et al., 2010), por analizar la estructura productiva de una economía y su impacto en el consumo eléctrico (Hang y Tu, 2007), por estudiar la evolución del precio de las distintas fuentes de energía (Feng et al., 2009), o por descomponer la estructura 
del consumo energético relacionándolo con el nivel de gasto público (Yuxiang y Chen, 2010).

Por otra parte, en los países avanzados se vienen observando medidas de política energética orientadas a potenciar el desarrollo y la innovación en las PYMES, medidas para la estandarización de procesos, o medidas para el desarrollo de tecnologías que permitan alcanzar economías de escala y la reducción de los costes de los equipos necesarios ante la implantación de sistemas de gestión de energía. Finalmente, también han surgido actuaciones encaminadas a acometer proyectos de integración de sistemas de gestión de energía que examinen tanto el lado de la oferta como el de la demanda. Toda esta estrategia pasa por la promoción de la investigación y el aumento de las capacidades de $\mathrm{I}+\mathrm{D}+\mathrm{i}$ en materia de eficiencia energética, potenciando el papel de los centros tecnológi$\cos$, las áreas de $\mathrm{I}+\mathrm{D}+\mathrm{i}$ y la investigación en universidades.

El análisis que proponemos en el siguiente apartado utiliza como marco conceptual el modelo empírico de crecimiento sostenible basado en la Curva de Kuznets Ambiental (CKA), ya utilizado por Cantos y Balsalobre (2011) para encontrar evidencia sobre la contribución de las fuentes renovables a la reducción de las emisiones contaminantes. Además, avanzamos, en la línea propuesta por Fisher-Vanden et al., (2004) $)^{15}$, en el análisis de la relación entre la intensidad energética y el esfuerzo público realizado en $\mathrm{I}+\mathrm{D}+\mathrm{i}$.

Figura 4

Evolución del precio de la electricidad y del déficit tarifario en España

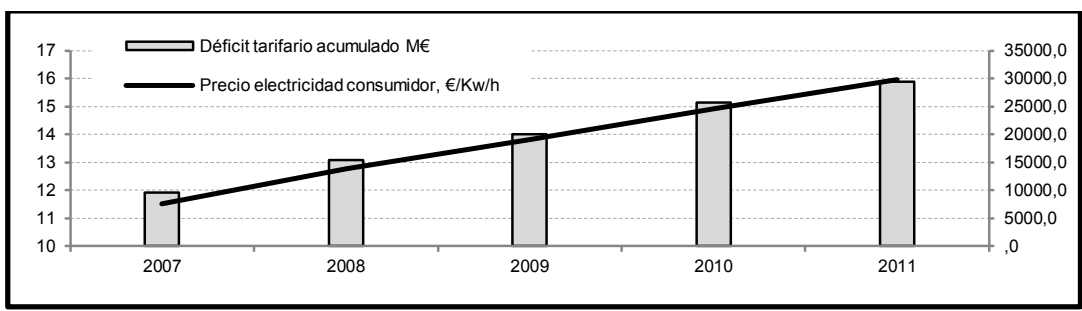

Nota: Eje principal (izda.): precio electricidad (sin impuestos), €/kw/h. Eje secundario (dcha.): déficit tarifario acumulado.

Fuente: UNESA.

Al observar los datos de la Figura 4, y teniendo en cuenta el elevado ritmo de implantación de las fuentes renovables en España a lo largo de los últimos años, es razonable formular la hipótesis de que el precio final de la energía

\footnotetext{
${ }^{15}$ Fisher-Vanden et al., (2004) demuestran que el esfuerzo innovador contribuye a la reducción de la intensidad energética en el contexto empresarial; algo que a nivel más general había sido apuntado por Porter y Van Der Linde (1995).
} 
eléctrica guarda cierta relación positiva con la creciente importancia de las fuentes renovables en el mix energético, donde los costes unitarios son muy superiores a los de las fuentes tradicionales (Agostini y Padilla, 2010). Por otro lado, también cabe esperar que, a corto plazo, el conjunto de medidas de apoyo a la mejora de la eficiencia energética guarde una relación positiva con el precio final de la energía. Y ello a pesar de que, tanto para el caso de la sustitución de fuentes como para el de mejora de la eficiencia, no todos los costes han sido trasladados a los pecios finales, como pone de manifiesto la persistente presencia de los déficits de tarifa durante más de una década. Por tanto, tal y como apunta Becker (2011), sería preciso que la política energética se enfocara con una mayor horizontalidad, ya que aspectos como las características del modelo productivo o los hábitos de consumo de usuarios domésticos y empresas, condicionan el sistema energético.

A pesar de la importancia que se ha venido concediendo al sector energético y a la problemática del cambio climático dentro del Plan Nacional de $\mathrm{I}+\mathrm{D}+\mathrm{i}$, resulta notoria la insuficiente planificación de la que ha adolecido la política energética nacional (IEA, 2009a), lo cual queda reflejado en los datos de un $\mathrm{I}+\mathrm{D}+\mathrm{i}$ energético cuyo peso en el PIB se mantiene constante en términos reales desde 1993 (ver Figura 1.1). Situación que contrasta con la elevada dedicación de recursos públicos a la promoción de las energías renovables que, junto con la nuclear, vienen acaparando en torno al 75\% del total de recursos públicos destinados a la investigación en tecnologías energéticas durante los últimos años. Esta deficiencia ha sido objeto de atención por parte de la Agencia Internacional de la Energía (AIE), que ha sugerido a España la necesidad de destinar una mayor cuantía de recursos a la investigación en aquellas áreas donde se presentan unas mayores necesidades de mejora en la eficiencia energética, tales como el sector del transporte (IEA, 2009a:137-144). En este sentido, toda intervención pública dirigida a corregir esas deviaciones con éxito, mejoraría el bienestar social neto siempre que los costes en que se incurre puedan ser absorbidos por las ganancias de bienestar obtenidas (Gillingham y Sweeney, 2010:128).

Además de que, en el plano teórico, parece fuera de toda duda la relación entre la $\mathrm{I}+\mathrm{D}+\mathrm{i}$ y la eficiencia energética, existen varios trabajos que han encontrado evidencia empírica. Por ejemplo, en Ruiz (2010) se ha encontrado evidencia de relación causal positiva entre los recursos públicos dedicados a la investigación en energía y la mejora en la intensidad energética, refrendando la hipótesis que subyace en este tipo de políticas y, por tanto, la eficacia de la intervención pública. Es sabido que en presencia de un fallo de mercado, los beneficios privados de una inversión en $\mathrm{I}+\mathrm{D}+\mathrm{i}$ estarían por debajo de los beneficios sociales, y una adecuada intervención pública contribuiría a alcanzar el óptimo social (Arrow, 1962; Griliches, 1992). En esta misma dirección, Fisher-Vanden et al., (2004) también han demostrado que el gasto en $\mathrm{I}+\mathrm{D}+\mathrm{i}$ es 
un factor que contribuye de manera importante a la reducción de la intensidad energética empresarial.

\section{APLICACIÓN DEL MODELO CKA AL GASTO PÚBLICO ENERGÉTICO}

Diversos estudios empíricos han puesto de manifiesto que se produce una interacción entre el crecimiento económico y la contaminación, de manera que la degradación medioambiental es una función, en forma de U-invertida, creciente hasta un determinado nivel de renta crítico, a partir del cual mejoras operadas en el nivel de renta se asocian, a su vez, con mejoras en el nivel de calidad ambiental (Grossman y Krueger, 1991), en lo que ha venido a conocerse como la Curva de Kuznets Ambiental (CKA). Con esta aportación pionera se inicia el estudio empírico de la relación entre el crecimiento económico y la degradación medioambiental, dando lugar a diversos trabajos posteriores que han aportado evidencia empírica, tanto a favor como en contra, sobre la CKA. En este sentido, la hipótesis de la CKA presenta ciertas características que le confieren ventaja en el estudio empírico de la relación entre el crecimiento económico y la degradación medioambiental (De Castro, 2009:106; Cantos y Balsalobre, 2011).

Figura 5

Curva de Kuznets Ambiental (CKA)

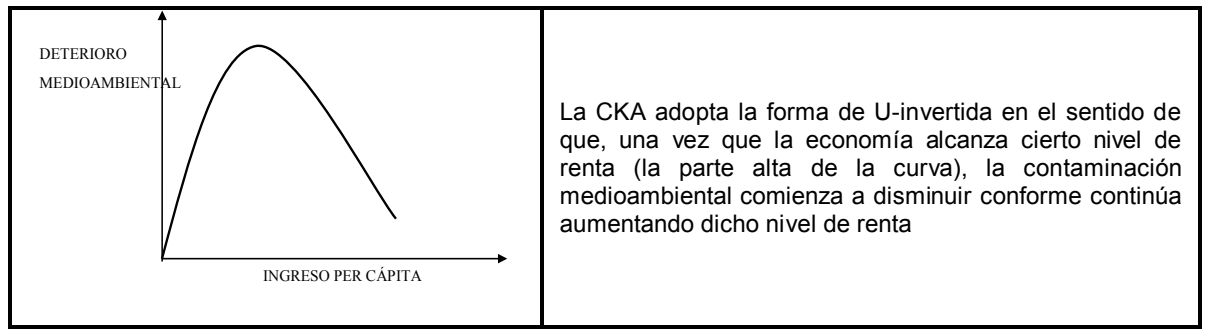

Fuente: Elaboración propia.

A partir de la exposición que se realiza en Grossman y Krueger (1991), la expresión generalizada de la Curva de Kuznets Ambiental puede establecerse como:

$$
G E I_{P C, t}=\alpha_{t}+\beta_{1} Y_{t}+\beta_{2} Y 2_{t}+\beta_{3} Z_{t}+e_{t}
$$

${ }^{16} G E I_{P C, t}$ representa el nivel de emisiones de gases efecto invernadero. El subíndice $i$ señala el país o región, $t$ indica el momento del tiempo, $\alpha$ es el promedio de presión ambiental cuando el ingreso no tiene especial relevancia sobre dicha variable, $\boldsymbol{\beta}$ representa la importancia relativa de las variables exógenas, $Y_{t}$ es el nivel de ingreso, $Z_{t}$ permite incorporar otras variables de influencia sobre la presión ambiental, mientras que $e_{t}$ es el término de error, normalmente distri- 
De acuerdo con el modelo propuesto por Andreoni y Levinson (1998), resulta especialmente significativa la vinculación que la CKA mantiene con el desarrollo tecnológico y con las mejoras medioambientales. El propio modelo desagrega el efecto total de la reducción de la contaminación medioambiental en tres componentes: un efecto de escala, que recoge un aumento en los niveles de polución conforme aumenta la actividad; un efecto composición, que se explica a través de la evolución o tercerización de las economías, de modo que este efecto generaría la forma de U-invertida de la curva; y, por último, un efecto progreso tecnológico y de eliminación de polución, en el sentido de que, a medida que aumenta la renta, se produce una disminución de la contaminación medioambiental como consecuencia de la aplicación de mejoras tecnológicas.

Sin embargo, conviene matizar que el crecimiento económico no resolverá, por sí solo, los problemas de contaminación puesto que, en ausencia de regulación ambiental, la trayectoria descrita por la relación "nivel de ingresos/contaminación", aun teniendo forma de U-invertida, puede llevar a que se alcance el tramo descendente de la curva (reducción de la contaminación) para un nivel de ingresos excesivo e innecesariamente elevado: teóricamente, esto se traduce en que el modelo no establece límite alguno en el nivel de ingresos necesario para generar la reducción de la contaminación. De este modo, el modelo CKA, no solo resulta compatible con la incorporación de medidas de regulación medioambiental, sino que el uso de variables explicativas, como las tecnologías de descontaminación, adquiere un gran valor interpretativo a la hora de interpretar el fenómeno descrito.

La hipótesis de la CKA asume que, conforme los países son económica y socialmente más avanzados, conceden una mayor importancia a las actividades de reciclaje y eliminación de residuos y que existen cambios paulatinos en las economías que modifican conductas medioambientales (p.e., el propio proceso de terciarización de las economías conduce a un proceso de reducción en el nivel de emisiones GEI). Estos procesos de cambio guardan relación con el tipo de políticas medioambientales utilizadas, con los niveles de educación ciudadana, con el grado de protección de los derechos de propiedad privada y con otras acciones que adoptan los países para mejorar su calidad medioambiental conforme aumentan su nivel de desarrollo. Finalmente, y a pesar de que queda más alejado de nuestra preocupación en este trabajo, hay que señalar que la relocalización internacional de las actividades contaminantes también tiene cabida en el modelo CKA.

Puesto que existe una necesidad, difícilmente soslayable, de utilizar la forma reducida del modelo CKA en el contraste empírico, no siempre es posible ex-

buido. Así pues, la anterior expresión permite identificar distintas formas de la relación entre medio ambiente y nivel de ingresos, así como los comportamientos inducidos al incorporar otras variables. 
plicar con claridad los coeficientes obtenidos al producirse relaciones de causalidad indirecta, en lo que constituye un "eslabón perdido" de esta metodología que bien podría acotarse analizando tres tipos de impactos sobre las emisiones: la regulación ambiental, el aporte tecnológico y la composición industrial (Grossman y Krueger, 1995). A partir de esta proposición, Torras y Boyce (1998) concluyen que es la regulación ambiental el componente más importante para acelerar un cambio tecnológico capaz de reducir el nivel de contaminación.

No obstante, en Grossman y Krueger (1995) se considera que el efecto "composición industrial" podría ser debido a que un ingreso per cápita creciente ("efecto de escala") puede venir acompañado de una reducción en la intensidad marginal de la contaminación del producto, siempre que los sectores más intensivos en contaminación se contraigan, ya que, en caso contrario, no podrá contrarrestarse el efecto de escala del impacto ambiental resultante del mayor nivel de producto agregado. Ahora bien, puede suceder que el efecto tecnológico sobrepase al efecto de escala cuando los sectores contaminantes produzcan bienes inferiores, lo que va a provocar que el consumo caiga con el aumento del ingreso, o bien, que se produzca una sustitución por importaciones (Panayotou, 1997).

Ha sido la teoría endogenista la que más ha puesto de manifiesto el impacto del progreso tecnológico sobre el comportamiento de la relación "nivel de ingresos/medio ambiente", debido a la mejora que experimentan los procesos de producción, incrementando la capacidad de sustitución de los recursos altamente contaminantes o degradantes por otros más ecológicos (Steger, 1996; Stokey, 1998, entre otros). Estos modelos defienden que una sociedad comprometida con la protección medioambiental está capacitada para invertir una cantidad de recursos cada vez mayor en su protección a medida que aumenta la renta. Si la contaminación disminuye con el incremento en el nivel de ingresos, el efecto tecnológico jugará un papel importante, de modo que la contaminación total disminuirá sistemáticamente a medida que el ingreso per cápita aumente, lo que explicaría que la causa fuese la "innovación inducida", en el sentido de Hicks. En resumen, dentro de la hipótesis de la CKA aparece implícitamente una teoría de la "innovación inducida" que apunta que, a medida que se incrementa el nivel de ingreso, las sociedades se encuentran más capacitadas para compensar los fallos de mercado. Además, Andreoni y Levinson (1998) consideran que, aun suponiendo que el medio ambiente fuese un bien normal donde los individuos maximizan su nivel de utilidad, la hipótesis de la CKA es razonable como consecuencia de la propia naturaleza de las tecnologías de reducción de contaminación ambiental, de modo que el cumplimiento de la CKA depende, fundamentalmente, de la tecnología, más que de las externalidades ambientales inherentes al crecimiento económico. 
Al objeto de seguir un orden lógico en la aplicación del modelo, estimaremos ahora la relación entre el nivel de emisiones de gases de efecto invernadero $\left(\mathrm{GEI}_{\mathrm{pc}}\right)$ y el gasto público en $\mathrm{I}+\mathrm{D}+\mathrm{i}$ energético que se ha venido realizando en España desde la década de los noventa. A continuación, se llevará a cabo una desagregación del gasto total en $\mathrm{I}+\mathrm{D}+\mathrm{i}$ energético, utilizando por separado el $\mathrm{I}+\mathrm{D}+\mathrm{i}$ en eficiencia energética y el gasto en $\mathrm{I}+\mathrm{D}+\mathrm{i}$ realizado en fuentes renovables. En este trabajo tomamos como referencia la metodología y los resultados obtenidos en Cantos y Balsalobre (2011), donde se analiza la relación entre el crecimiento económico -en términos de PIB per cápita- y un patrón de consumo energético que enfrenta el consumo energético de procedencia renovable con el consumo energético de fuentes convencionales, encontrando evidencia de una contribución de las medidas de promoción de las energías renovables sobre la reducción de la variable $\mathrm{GEI}_{\mathrm{pc}}$ en España.

Una vez que analizamos la aportación del esfuerzo tecnológico conjunto y por separado, estudiamos si los resultados son coherentes con el comportamiento esperado. El punto donde la función que relaciona el nivel de renta con la contaminación se vuelve decreciente, representaría el nivel de renta crítico en el que se quiebra la relación creciente entre renta y contaminación, dentro del tramo relevante. Cuando se introducen, de forma alternativa, otras variables explicativas, el punto de quiebra se ve afectado por los cambios introducidos, reflejando la contribución diferencial de cada variable al resultado, teniendo en cuenta que cuanto mayor sea el nivel de renta crítico (el punto de quiebra) más ineficaces resulta el conjunto de medidas de regulación energética que se esconden detrás de la variable. Nótese que, por construcción, el modelo no puede aportar resultados estrictos sobre la eficiencia de las medidas que se reflejan en las variables, puesto que ninguna incluye precios, sino tan solo cantidades. Sin embargo, estos resultados pueden utilizarse para completar razonamientos fuera de este marco analítico. Las variables utilizadas y las fuentes de las que proceden, son las siguientes:

Tabla 1

Variables utilizadas en el modelo

(en millones de euros de 2010)

\begin{tabular}{|l|l|}
\hline \multicolumn{2}{|l|}{ Variable endógena: } \\
\hline$G E I_{P C, t}$ & $\begin{array}{l}\text { Emisión gases efecto invernadero para el periodo 1992-2008 }{ }^{17} \text {. En toneladas } \\
\text { equivalentes de } \mathrm{CO}_{2} . \text { Fuente: Ministerio de Medio Ambiente (MMA). }\end{array}$ \\
\hline
\end{tabular}

${ }^{17}$ Se utiliza la serie histórica hasta 2008 a dos razones. Por un lado, con el objeto de poder comparar directamente los resultados con los obtenidos en Cantos y Balsalobre (2011). Por otro, se trata de evitar los efectos no deseados de la profunda y persistente caída del PIB durante los años de la crisis económica, sobre el resultado de la estimación. 
Tabla 1 (continuación)

Variables utilizadas en el modelo

(en millones de euros de 2010)

\begin{tabular}{|l|l|}
\hline \multicolumn{2}{|l|}{ Variables independientes: } \\
\hline$P B_{P C, t}\left(€ /_{p c 2000}\right)$ & $\begin{array}{l}\text { Nivel de renta, en términos per cápita (euros a precios constantes del año 2000), } \\
1992-2008 . \text { Fuente: INE }\end{array}$ \\
\hline$P I B^{2}{ }_{P C, t}$ & $\begin{array}{l}\text { Validación de la forma funcional cuadrática en forma de U-invertida entre el nivel de } \\
\text { emisiones y el nivel de renta per cápita, 1992-2008 (Correa, 2007:22). Fuente: INE, }\end{array}$ \\
\hline$I+D+E n e r g t o t_{(t-2)}$ & $\begin{array}{l}\text { Gasto público en I+D+i en el sector energético en España (millones euros de 2010), } \\
\text { aplicando un retardo de dos períodos, a precios y tipo de cambio de 2010, en } \\
\text { términos per cápita (INE, 2012), 1992-2008. Fuente: AlE (2011) }\end{array}$ \\
\hline$I+D+i$ nuclear $(t-1)$ & $\begin{array}{l}\text { Gasto público en I+D+i en el sector energético en España (millones euros de 2010), } \\
\text { en energía nuclear, con retardo de un período, a precios y tipo de cambio de 2010, y } \\
\text { en términos per cápita, 1992-2008. Fuente: AlE (2011) }\end{array}$ \\
\hline
\end{tabular}

Fuente: Elaboración propia.

Figura 6

Distribución del I+D+I público por tecnología energética

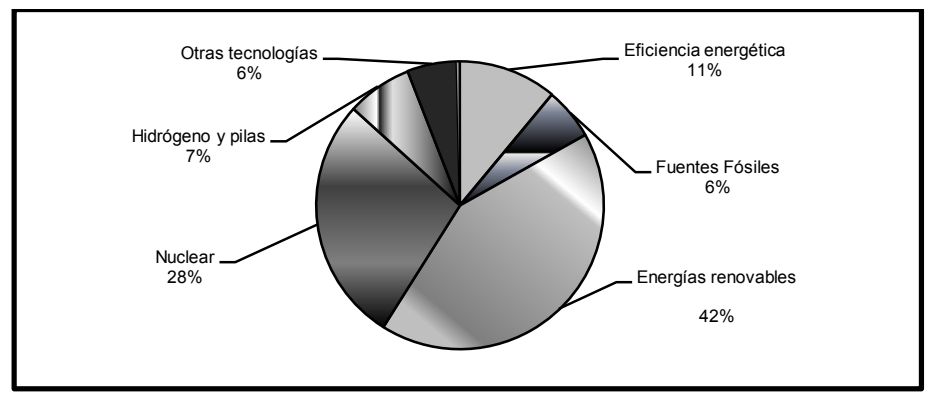

Fuente: "IEA Energy Technology R\&D Statistics, Database (2010), se desagrega, a nivel nacional, el presupuesto público en $\mathrm{I}+\mathrm{D}+\mathrm{i}$ destinado a eficiencia energética, energías renovables, nuclear, y el resto de actividades energéticas (fuentes fósiles y otros).

\section{ESTIMACIÓN DEL MODELO Y DISCUSIÓN DE RESULTADOS}

Una primera versión del modelo (Tabla 2) permitirá calcular el punto de quiebra de la CKA, indicando los requerimientos de renta per cápita necesarios para que la sociedad empiece a experimentar una reducción en el nivel de GEI per cápita. Siguiendo la metodología habitual en muchos trabajos de este tipo, la estimación se lleva a cabo mediante una regresión lineal por mínimos cuadrados ordinarios (MCO), utilizando como variables independientes la renta per cápita a precios constantes de 2000 ( PIB $_{\mathrm{PC}}$ ), así como la expresión cuadrática de dicha 
variable (PIB2pc), responsable de la forma de U-invertida de la función principal (Correa, 2007:22) $)^{18}$.

Tabla 2

Modelos y resultados

\begin{tabular}{|c|c|c|c|}
\hline \multicolumn{4}{|c|}{$\begin{array}{l}\text { Modelo } 1: \mathrm{GEI}_{\mathrm{PC}, \mathrm{t}}=\alpha+\beta_{1} \mathrm{PIB}_{\mathrm{PC}, \mathrm{t}}+\beta_{2} \mathrm{PIB}^{2}{ }_{\mathrm{PC}, \mathrm{t}}+\varepsilon_{\mathrm{t}} \\
\text { Modelo } 2: \mathrm{GEI}_{\mathrm{PC}, \mathrm{t}}=\alpha+\beta_{1} \mathrm{PIB}_{\mathrm{PC}, \mathrm{t}}+\beta_{2} \mathrm{PIB}_{\mathrm{PC}, \mathrm{t}}+\beta_{3, \mathrm{t}} \mathrm{l}+\mathrm{D}+\mathrm{i} \text { enertot }(\mathrm{t}-2)+\varepsilon_{\mathrm{t}} \\
\text { Modelo 3: } \mathrm{GEI}_{\mathrm{PC}, \mathrm{t}}=\alpha+\beta_{1} \mathrm{PIB}_{\mathrm{PC}, \mathrm{t}}+\beta_{2} \mathrm{PIB}_{\mathrm{PC}, \mathrm{t}}+\beta_{4, \mathrm{t}}+\mathrm{D}+\mathrm{i} \text { nuclear(-1)+ }+\varepsilon_{\mathrm{t}}\end{array}$} \\
\hline \multicolumn{4}{|c|}{$\begin{array}{l}\text { Estimación usando las observaciones del periodo 1992-2008 (T=17). } \\
\text { Variable dependiente para todos los modelos: GEl }\end{array}$} \\
\hline & Modelo 1 & Modelo 2 & Modelo 3 \\
\hline Variable & Coeficiente(Desv.tip) & Coeficiente(Desv.tip) & Coeficiente(Desv.tip) \\
\hline Const & $-8,2459(4,99085)$ & $-7,4956(9,373)$ & $-13,7817(3,89651)$ \\
\hline $\mathrm{PIB}_{\mathrm{PC}, \mathrm{t}}\left(\beta_{1}\right)$ & $0,00190(0,00066)^{\star \star}$ & $0,00227(0,001)^{\star \star}$ & $0,00301(0,00053)^{\star \star \star}$ \\
\hline $\mathrm{PIB}^{2}{ }_{\mathrm{PC}, \mathrm{t}}\left(\beta_{2}\right)$ & $-5,04 e-08(2,18 e-08)^{\star \star}$ & $-6,90 e-08(2,9 e-08)^{\star *}$ & $-9,182 e-08(1,77989 e-08)^{\star \star *}$ \\
\hline $1+D+i$ enertot $(t-2)\left(\beta_{3}\right)$ & - & $-0,0249334(0,01)^{\star}$ & - \\
\hline $1+D+i$ nuclear $_{(t-1)}\left(\beta_{4}\right)$ & - & - & $-4,757 e-08(1,36971 \mathrm{e}-08)^{\star \star \star}$ \\
\hline R-cuadrado corregido & 0,832900 & 0,864346 & 0,919352 \\
\hline Durbin Watson & 0,890230 & 1,843900 & 1,782253 \\
\hline $\begin{array}{l}\text { Contraste de } \\
\text { heterocedasticidad de } \\
\text { White }\end{array}$ & $\begin{array}{c}\mathrm{P}(\text { Chi-Square }(5)>5,84118)= \\
0,321981 \text { ** }\end{array}$ & $\begin{array}{c}P(\text { Chi-Square }(9)>13,9698)= \\
0,123404 \text { ** }\end{array}$ & $\begin{array}{c}\mathrm{P}(\text { Chi-Square }(9)>7,21618)= \\
0,614623^{* *}\end{array}$ \\
\hline $\begin{array}{l}\text { Contraste de Chow de } \\
\text { cambio estructural en la } \\
\text { observación }\end{array}$ & $\begin{array}{c}P(F(3,11)>6,59856)= \\
0,00818746 \text { ** }\end{array}$ & $P(F(4,7)>1,70161)=0,252928$ ** & $\begin{array}{c}P(F(4,8)>2,85083)= \\
0,0968288^{* *}\end{array}$ \\
\hline $\begin{array}{l}\text { Contraste LM de } \\
\text { autocorrelación }\end{array}$ & $\begin{array}{c}\mathrm{P}(\mathrm{F}(1,13)>3,63968)= \\
0,0787508^{* *}\end{array}$ & $P(F(1,10)>0,240907)=0,63414$ ** & $\begin{array}{c}P(F(1,11)>0,0152965)= \\
0,903801^{*}\end{array}$ \\
\hline $\begin{array}{l}\text { Contraste de normalidad } \\
\text { de los residuos }\end{array}$ & $\begin{array}{l}\text { Chi-cuadrado }(2)=2,17332 \\
\text { con valor } p=0,337342^{* *}\end{array}$ & $\begin{array}{l}\text { Chi-cuadrado }(2)=0,37984, \text { con } \\
\text { valor } p=0,827025^{* *}\end{array}$ & $\begin{array}{l}\text { Chi-cuadrado }(2)=0,680179 \\
\text { con valor } p=0,711707^{\star *}\end{array}$ \\
\hline $\begin{array}{l}\left(-\beta_{1} / 2 \beta_{2}\right)=\text { punto de } \\
\text { quiebra CKA }\end{array}$ & $18.861,71 €$ & $16.483,14 €$ & $16.399,47 €$ \\
\hline
\end{tabular}

Nivel de confianza en los contrastes: $\left(^{* * *}\right)$ indica el 99\%, $\left({ }^{* *}\right)$ el $95 \%$ y $\left(^{*}\right)$ el $90 \%$

Fuente: Elaboración propia.

$\mathrm{Al}$ analizar los tres modelos estimados, los coeficientes $\beta_{1}$ y $\beta_{2}$ son significativos y, además, toman el signo esperado que confirma la forma de U-invertida de la función. Por otra parte, el coeficiente $\beta_{3}$ del modelo 2 resulta significativo y con el signo esperado, y lo mismo ocurre en el modelo 3 con $\beta_{4}$. Teniendo en cuenta que se ha utilizado un retardo de 2 periodos para la variable $\mathrm{I}+\mathrm{D}+\mathrm{i}$ enertot en el modelo 2 , y un retardo de un período en la variable $\mathrm{I}+\mathrm{D}+\mathrm{i}$ nuclear, ha mejorado la bondad general del ajuste en ambos modelos (medida por el R-cuadrado corregido). Como comentario adicional, advertimos que también ha sido estimado el modelo utilizando las variables de gasto de $\mathrm{I}+\mathrm{D}+\mathrm{i}$ en renovables, por un lado, y el mismo tipo de gasto en eficiencia energética, por

${ }^{18}$ La literatura empírica viene verificado el cumplimiento de la CKA a través de un modelo de regresión lineal estimado por MCO. Este método ha sido ampliamente utilizado para analizar la relación entre el crecimiento económico y la degradación medioambiental (Grosssman y Krueger, 1991; Andreoni y Levinson, 1998; Correa, 2004; Saravia, 2004, entre otros). 
otro, pero los coeficientes obtenidos en ambos casos no son aceptables para un nivel de confianza del $90 \%$. Finalmente, cabe mencionar que el contraste de estabilidad de parámetros de Chow toma como punto de ruptura el año 1999, y avala la estabilidad de los parámetros obtenidos en las estimaciones de los tres modelos.

Como se indica en la Tabla 2, en el modelo 1 el requerimiento de renta para que comience a corregirse el deterioro medioambiental parece considerablemente elevado $(18.861,71 €)$, cumpliéndose la forma de U-invertida de la función en los términos esperados $\left(\beta_{1}>0, \beta_{2}<0\right)$, con una bondad del ajuste (Rcuadrado corregido) de 0,8329 . En particular, la estimación sitúa el citado nivel de renta per cápita a partir del cual, ceteris paribus, las emisiones comienzan a retroceder, en un nivel que todavía no se había alcanzado en España en 2008, con los datos de la serie utilizada.

Por su parte, en el modelo 2 se incorpora a la estimación la variable gasto público en $\mathrm{I}+\mathrm{D}+\mathrm{i}$ energético $(\mathrm{I}+\mathrm{D}+\mathrm{i}$ enertot $(\mathrm{t-2})$ ), con el fin de poder comparar los resultados con los del modelo 1. Esta variable pretende capturar la contribución pública a la mejora de la eficacia, lo que debería tener su reflejo sobre la reducción de emisiones GEI. Resulta de interés saber si el modelo 2 mejora el requerimiento de renta per cápita para alcanzar la corrección medioambiental, en términos de $\mathrm{PIB}_{\mathrm{PC}}$. Para ello incorporamos al análisis la variable $I+D+i$ energtot $_{(t-2)}$, que representa el nivel de gasto público en $\mathrm{I}+\mathrm{D}+\mathrm{i}$ en el sector energético, con un retardo de dos años. La introducción de un retardo en la variable permite capturar el lapso temporal necesario para que el impacto del gasto con esta finalidad tenga su reflejo en la tecnología aplicada y, finalmente, en el nivel de las emisiones contaminantes, puesto que la incorporación tecnológica no se produce de manera inmediata. El número de años de retardo que mejor se ha comportado en el ajuste ha sido dos.

La estimación del modelo 2 también cumple con los test realizados, a la vez que ve aumentada la bondad del ajuste medido por el R-cuadrado corregido (hasta 0,8643$)$, y responde también a la forma de U-invertida de la CKA $\left(\beta_{1}>0\right.$, $\left.\beta_{2}<0\right)$. En este caso, el nivel de renta per cápita necesario para que se dé un techo en las emisiones contaminantes $\left(\mathrm{GEI}_{\mathrm{PC}}\right)$ se sitúa en los 16.483,14 euros, cifra sensiblemente inferior a la que se daría en ausencia de la variable $I+D+i$ energtot $_{(t-2)}$. El análisis del resultado se sujeta a las limitaciones de cualquier análisis parcial y debe interpretarse en el sentido de que el esfuerzo en $\mathrm{I}+\mathrm{D}+\mathrm{i}$ público se asocia positivamente con la reducción de la contaminación, tal y como apunta el signo negativo de $\beta_{3}$, siendo más importantes las diferencias relativas entre los valores obtenidos de renta per cápita, que las absolutas. Es suficiente con interpretar la aportación positiva de la variable a la corrección ambiental y su eficacia al lograr reducir los requerimientos de renta para que se frene el ascenso de las emisiones contaminantes. 
Tal y como se ha señalado en el apartado anterior de este trabajo, ante los objetivos generales de la política energética representados por el control de las emisiones contaminantes, la reducción de la dependencia y el logro de un mercado energético español más eficiente, resulta fácil observar que la prioridad de la política energética española se ha centrado, en mayor medida, en el objetivo intermedio de la sustitución de fuentes contaminantes por otras renovables y más limpias, para lo que se han movilizado importantes cantidades de recursos públicos con este fin. Sin embargo, nuestro interés en este trabajo gira sobre el gasto público para fomentar la mejora tecnológica en el sistema energético, ingrediente básico de las ganancias en eficiencia que se pretenden alcanzar.

Por eso, nuestro siguiente paso consiste en estimar el modelo 3, donde se sustituye la variable utilizada que recoge la aportación pública total a la $\mathrm{I}+\mathrm{D}+\mathrm{i}$ energética $\left(I+D+i\right.$ enertot $\left._{(t-2)}\right)$, por otra que recoge tan solo la aportación pública a la $\mathrm{I}+\mathrm{D}+\mathrm{i}$ vinculada a la energía nuclear $\left(I+D+i\right.$ nuclear $\left._{(t-1)}\right)$, donde utilizaremos el retardo de un año. Como se observa en la Tabla 2, los resultados del ajuste realizado para el modelo 3 incluyen una mejora en el R-cuadrado corregido (se incrementa hasta 0,9193 ), mientras que los test realizados siguen otorgando validez estadística. En este caso, el punto de quiebra de la renta per cápita, esto es, el nivel de renta necesario para que las emisiones de gases comiencen a descender, es de 16.399,47 euros, cifra inferior a la que se daría bajo la hipótesis de no existir ninguna acción de corrección medioambiental (la correspondiente al modelo 1), y los signos de los coeficientes $\beta$ estimados son los esperados, en el sentido de que la contribución de la variable $I+D+i$ nuclear $_{(t-1)}$ a la corrección ambiental es positiva. La interpretación conjunta de los resultados correspondientes a los tres modelos permite destacar, además de la mejora en la calidad del ajuste que se obtiene en el modelo 3, el hecho de que el umbral de renta obtenido en éste último es inferior al del modelo 2. Este hallazgo lo interpretamos en el sentido de que, no solo es eficaz el apoyo tecnológico público a la energía nuclear, sino que su eficacia es superior a la media del gasto público en $\mathrm{I}+\mathrm{D}+\mathrm{i}$, puesto que el nivel de renta (y el tiempo) necesario para frenar las emisiones de gases de efecto invernadero, es inferior al obtenido en el ajuste del modelo 2.

La Figura 7 permite visualizar los resultados conjuntos de los tres modelos que han sido estimados, por lo que se refiere al punto de quiebra obtenido, en cada caso, y al consecuente requerimiento de renta per cápita necesario para invertir la tendencia creciente de las emisiones de gases de efecto invernadero. Precisamente, la renta per cápita necesaria para que se produzca la quiebra del ritmo ascendente de dichas emisiones será más elevada en ausencia de medidas correctoras (M_1) que cuando se cuenta con alguna de ellas, lo que se traduce en un mayor stock de contaminación y en un mayor tiempo para conseguirlo. La diferencia existente en el requerimiento de renta para M_1 y para cualquiera de los otros dos resultados, es considerable, confirmando la hipótesis de que existe 
una relación significativa, y de signo esperado, entre el esfuerzo realizado en corrección ambiental mediante el conjunto de políticas de I+D+i (M_2), y la contención de las emisiones contaminantes. Ahora bien, cuando se utilizan datos de gasto público en $\mathrm{I}+\mathrm{D}+\mathrm{i}$ vinculados a energía nuclear (M_3), puede apreciarse que la eficacia de las medidas de corrección ambiental es ligeramente superior a la obtenida en $M_{-}$2, al alcanzarse el punto de quiebra de las emisiones con una renta per cápita inferior.

En efecto, en la medida en que el punto de quiebra calculado en M_2 se produce para un nivel de renta per cápita superior al nivel correspondiente al punto de quiebra obtenido en M_3, puede interpretarse en términos económicos como una ventaja asociada al uso del gasto en $\mathrm{I}+\mathrm{D}+\mathrm{i}$ en energía de fuente nuclear, en relación a la media del conjunto de gasto en $\mathrm{I}+\mathrm{D}+\mathrm{i}$ energético. Dicha ventaja tiene que ver con el menor tiempo necesario para alcanzar la fase descendente del nivel de emisiones contaminantes, lo que se produciría bajo el supuesto general de que los países observan en el tiempo un ritmo creciente de renta per cápita. Puesto que uno de los principales objetivos de la política energética a largo plazo es reducir el nivel de emisiones contaminantes a la atmósfera, una medida adoptada será superior a otra si logra alcanzar el objetivo en el menor tiempo posible, puesto que menores serán las penalizaciones que el Estado tendrá que asumir por el incumplimiento de los objetivos de emisiones que la Unión Europea asigna a cada Estado miembro, como consecuencia de los compromisos adquiridos en el Acuerdo de Kioto ${ }^{19}$.

Figura 7

Estimaciones del modelo CKA para cada una de las variantes utilizada

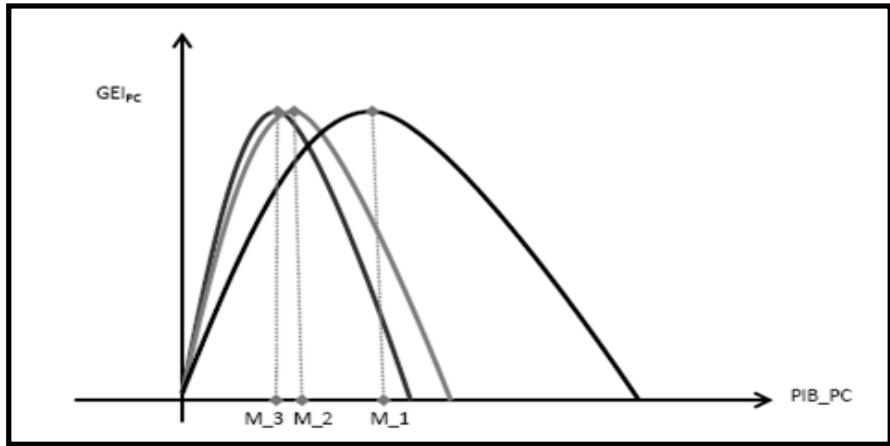

Notas: M1:modelo1; punto quiebra PIB 18.861,71€; M2: modelo2; punto de quiebra en PIB 16.483,14€; M3: modelo 3; punto de quiebra en PIB 16.399, $47 €$

Fuente: Elaboración propia.

${ }^{19}$ Para un mayor detalle sobre el cálculo del punto de quiebra, véase Selden y Song (1994), Grossman Krueger (1995), o Correa (2007). 
También podemos comparar los resultados aquí obtenidos con los alcanzados en Cantos y Balsalobre (2011), por lo que se refiere al punto de quiebra de la función. En dicho trabajo se relacionaba el nivel de emisiones $\mathrm{GEI}_{\mathrm{PC}}$ con la incorporación de una variable denominada PATROCEN, que representaba el cambio en el patrón de consumo energético nacional derivado del incremento en el peso específico de las energías renovables dentro del mix energético español. El nivel de renta correspondiente al punto de quiebra que se obtuvo ascendía a 18.054 euros, mientras que nuestro modelo M_2, sitúa al punto de quiebra, como se ha visto, en el equivalente a una renta per cápita de 16.483 euros, cifra inferior a la entonces obtenida, aunque aquélla ecuación incluía otras diferen$\operatorname{cias}^{20}$. A pesar de que no se puede llevar a cabo una comparación plenamente válida entre ambos modelos, nos permite apuntar la hipótesis de que existe una cierta superioridad, en relación con la eficacia de la política energética aplicada, de las medidas de corrección ambiental basadas en el gasto público en $\mathrm{I}+\mathrm{D}+\mathrm{i}$ energético, en relación a las medidas basadas en el fomento de fuentes energéticas renovables.

A la luz de los resultados obtenidos, conviene hacer dos precisiones para establecer el alcance de las interpretaciones derivadas de los mismos. En primer lugar, la escasa cuantía de la $\mathrm{I}+\mathrm{D}+\mathrm{i}$ pública aplicada al programa de mejora de la eficiencia energética, ya pone una limitación importante a la interpretación que pueda darse a partir del ajuste estadístico de datos, y ello al margen de que tampoco queda determinada la relación causal entre las variables. De hecho, ni siquiera ha podido obtenerse una relación estadísticamente significativa cuando hemos utilizado algunos componentes del gasto público en $\mathrm{I}+\mathrm{D}+\mathrm{i}$, como el aplicado a la mejora de la eficiencia energética o el aplicado a la energía de fuentes fósiles, probablemente debido a su escasa importancia cuantitativa (véase Figura 7). Estas limitaciones invitan a utilizar los resultados obtenidos con mucha cautela.

En segundo lugar, son conocidos los problemas asociados a la medición de los efectos nocivos que puede provocar la producción de energía eléctrica de fuente nuclear, dado que no existe un método generalmente aceptado para traducir, en términos de coste-beneficio, el riesgo de accidente en la fase de producción y, sobre todo, en la fase de almacenamiento de los residuos. En todo caso, la variable más comúnmente utilizada para medir la contaminación ambiental es la emisión de $\mathrm{CO}_{2}$ a la atmósfera en términos de flujo, quedando

${ }^{20} \mathrm{La}$ ecuación que se estimó es la siguiente: $\mathrm{GEI}_{\mathrm{PCt}}=\alpha+\beta_{1} \mathrm{PIB}_{\mathrm{pc}, \mathrm{t}}+\beta_{2} \mathrm{PIB}_{\mathrm{pc}, \mathrm{t}}^{2}+\beta_{3} \mathrm{GINI}_{\mathrm{t}}+$ $\beta_{4}$ PATROCEN ${ }_{t}+\varepsilon_{t}$, donde PATROCEN es, como ya se ha dicho, una variable que recoge el patrón de consumo energético que diferencia entre energía procedente de fuentes renovables y no renovables, y donde GINI es una variable que representa un índice de desigualdad económica introducida con el fin de obtener evidencia de su contribución a la corrección ambiental. 
fuera del modelo cualquier otra fuente de contaminación ambiental, como pudiera ser la provocada por el riesgo de accidente nuclear. Además, la diferencia obtenida a favor del gasto público en $\mathrm{I}+\mathrm{D}+\mathrm{i}$ vinculado a esta fuente energética, en relación a la media de este tipo de gasto, no es suficientemente importante como para afirmar inequívocamente su superioridad, aspecto que deberá ser corroborado por posteriores trabajos.

\section{CONCLUSIONES}

Para un país como España que cuenta con un importante nivel de desarrollo económico en el concierto mundial, el mantenimiento de una tasa de crecimiento compatible con una reducida tasa de desempleo, y compatible, a su vez, con una contención de las emisiones de gases contaminantes, pone inevitablemente el acento en la necesidad, tanto de buscar una sustitución de fuentes energéticas fósiles en pro de unos patrones de consumo menos nocivos, como de reducir la intensidad del consumo energético. Durante la última década, el gasto público asociado con las políticas energéticas ha estado muy sesgado hacia la promoción de fuentes renovables, pero se ha plasmado muy mayoritariamente mediante la fijación de precios a la producción muy superiores a los de mercado, en detrimento de la promoción directa de la innovación tecnológica en el ámbito del uso final de esa energía para fines de producción intermedia de bienes y servicios energéticamente más eficientes.

Además del escaso aporte directo del presupuesto público a la $\mathrm{I}+\mathrm{D}+\mathrm{i}$ energética, tampoco puede decirse que esta partida de gasto haya manifestado su preferencia por las medidas de mejora de la eficiencia energética, a pesar de que el índice español de intensidad energética aleja a España de la media europea y de los países más avanzados (Mendiluce et al., (2010) y IEA (2012). Aunque en las economías desarrolladas se viene observando una disminución progresiva de los recursos públicos destinados a la $\mathrm{I}+\mathrm{D}+\mathrm{i}$ en el sector de la energía, también es cierto que esta tendencia ha discurrido de forma paralela a la reducción de sus niveles de intensidad energética (Ruiz, 2010), cosa que no ha ocurrido en España. Con todo, los problemas derivados de un persistente déficit de atención pública a la $\mathrm{I}+\mathrm{D}+\mathrm{i}$ energética no acaban ahí, sino que, como ya señalaran Mansfield y Switzer (1984), simultáneamente desincentivan la inversión adicional y complementaria del sector privado en esta misma dirección.

De forma algo diferente a lo llevado a cabo en algunas referencias de la literatura económica, donde se vincula la evolución de las emisiones de gases de efecto invernadero con la intensidad energética (Kaya, 1989; Alcántara y Roca, 1995), en el presente trabajo hemos adoptado una variante en relación a dichos estudios, aplicando una versión cuadrática del modelo de la Curva de Kuznets Ambiental (CKA) para estudiar dos aspectos: primero, si hay una relación significativa entre el esfuerzo de la $\mathrm{I}+\mathrm{D}+\mathrm{i}$ pública realizada en España y las emisio- 
nes de contaminantes a la atmosfera medidas por el $\mathrm{CO}_{2}$; segundo, cual es el nivel de renta per cápita a partir del cual se invertiría la tendencia alcista de las emisiones contaminantes. En cuanto al primero, en las tres estimaciones realizadas hemos podido obtener evidencia de que hay una relación negativa y significativa entre los distintos componentes de la $\mathrm{I}+\mathrm{D}+\mathrm{i}$ pública utilizados, y el nivel de emisiones de $\mathrm{CO}_{2}$ a la atmósfera, lo que debe interpretarse en el sentido de que el gasto público en investigación energética se asocia indudablemente con menores emisiones contaminantes.

Los resultados obtenidos apoyan la hipótesis de que el gasto público en $\mathrm{I}+\mathrm{D}+\mathrm{i}$ ha contribuido positivamente a la reducción de las emisiones de $\mathrm{CO}_{2}$ a la atmósfera, obteniendo evidencia de que dicho gasto enfocado hacia la energía de fuente nuclear habría tenido una ligera ventaja en relación a la media del gasto público realizado con esta finalidad. Además, ante el objetivo de reducir las emisiones de gases contaminantes, el gasto público en $\mathrm{I}+\mathrm{D}+\mathrm{i}$ habría sido algo más eficaz que la sustitución de fuentes energéticas tradicionales por fuentes renovables. Sin embargo, el análisis realizado no permite comparaciones en términos de eficiencia, puesto que no incorpora información alguna sobre costes (el gasto público es solo una pequeña parte de la $\mathrm{I}+\mathrm{D}+\mathrm{i}$ ), si bien, no sería muy arriesgado formular una hipótesis a favor de la mayor eficiencia del gasto público en $\mathrm{I}+\mathrm{D}+\mathrm{i}$ energético en relación al gasto aplicado a la promoción de fuentes renovables.

\section{REFERENCIAS BIBLIOGRÁFICAS}

AGOSTINI, L.; y PADILLA, J. (2010): La promoción de las energías renovables: La experiencia española, Electricidad Verde: Energías Renovables y Sistema Eléctrico, pp. 517-541. MOSELLE B.; PADILLA, J.; y SCHMALENSEE, R. (eds.), Marcial Pons, Madrid, Barcelona, Buenos Aires.

ALCÁNTARA, V.; y ROCA, J. (1995). "Energy and CO2 of analysis and some results for 1980-90", Energy Economics, n. 17(3), pp. 221-230.

ALFRANCA, O. (2007): "Política fiscal, crecimiento económico y medio ambiente", Revista de economía, ICE., n. 835, pp.77-93.

ALFRANCA, O. (2009): "Regulación ambiental e innovación", Clm.economía: Revista económica de Castilla - La Mancha, n. 15, pp. 9-31.

ANDREONI, J.; y LEVINSON, A. (1998): The Simple Analytics of the Environmental Kuznets Curve, NBER Working Papers, n. 6739.

ARROW, K. (1962): "Economic Welfare and the Allocation of Resources to Invention," in R.R. Nelson (ed.), The Rate and Direction of Economic Activity, Princeton University Press, N.Y 
ARROW, K.; BOLÍN, B.; CONSTANZA, R.; DASGUPTA, P.; FOLKE, C.; HOLLING, C.; JANSSON, B.; LEVIN, S.;MÄLER, K.; PERRINGS C.; y PIMENTEL, D. (1995): "Economic growth carrying capacity, and the environmental", Science n. 268, pp. 520-521

BALDWIN, R. (1995): “Does sustainability require growth?" Goldin, I. \& Winters, L.A. (eds.), The economics of sustainable development, Cambridge University Press, Cambridge

BECKLER, F (2011): "Prospectiva de la electricidad en España", Estudios de Economía Aplicada, vol.29-2, pp.415-417.

CANTOS, J.M.; y BALSALOBRE, D. (2011): "Las energías renovables en la Curva de Kuznets Ambiental: Una aplicación para España", Estudios de Economía Aplicada, vol.29-2, (32 pp.).

CAPÓ, J. (2009): Curva de Kuznets Ambiental: evidencia para Europa, Documento de trabajo, Universidad des Illes Balears.

CORREA, F. (2007): Crecimiento económico, desigualdad social y medio ambiente: evidencia empírica para América Latina, Grupo de Economía Ambiental. Universidad de Medellín.

CORREA, F. (2007):"Crecimiento económico y medio ambiente: Una revisión analítica de la hipótesis de la curva ambiental de Kuznets" Grupo de Economía Ambiental. Universidad de Medellín.

CORREA, F.; Grupo de Economía Ambiental-GEA (2004): "Crecimiento económico y medio ambiente: una revisión analítica de la hipótesis de la Curva Ambiental de Kuznets". Semestre Económico, núm. Julio-Diciembre, pp. 73104.

DE BRUYN, (2000): "Economic growth and the environment: an empirical analysis"; The Netherlands Kluwer Academic Publishers.

DE CASTRO, L.M. (2009): "Crecimiento Económico y Medioambiente", Economía y Medio Ambiente, n. 847, pp. 93-110.

DEL RÍO, P.; LABANDEIRA, X.; y LINARES, P. (2009): "La interacción del sistema europeo de comercio de emisiones con otros instrumentos de política", Papeles de Economía Española, n. 121, pp. 211-223.

ENERCLUB (2009): Primer análisis del estado de la innovación en el área de las tecnologías energéticas en España, Club Español de la Energía, Madrid.

FENG, T.; SUN, L.; y ZHANG, Y. (2009): "The relationship between energy consumption structure, economic structure and energy intensity in China", Energy Policy, n.37, pp. 5.475-5.483.

FERNÁNDEZ, P. (2007): "La contribución de la ciencia, la tecnología y la innovación a la sostenibilidad de la energía en la UE”, en GARCÍA DELGADO, J. L.; y JIMÉNEZ, J. C. (Drs.): Energía y Regulación, Thomson-Civitas, Madrid.

FISHER-VANDEN, K.; JEFFERSON, G.H.; LIU, H.; y TAO, Q. (2004): "What is driving China's decline in energy intensity? Resource and Energy Economics, 26, pp. 77-97. 
FORO NUCLEAR (2012): Energía 2012, Madrid.

GILLINGHAM, K.; y SWEENEY, J. (2010): "Fallos de mercado y la estructura de las Externalidades", Electricidad Verde:Energías Renovables y Sistema Eléctrico, pp. 127-166. MOSELLE B.; PADILLA, J.; y SCHMALENSEE, R. (eds.), Marcial Pons, Madrid, Barcelona, Buenos Aires.

GILLINGHAM, R.; NEWELL, G.; PALMER, K. (2009): "Energy Efficiency Economics and Policy," Annual Review of Resource Economics, Annual Reviews, vol. 1,n.1, pp. 597-620.

GITLI, E.; y HERNÁNDEZ, G. (2002): La existencia de la curva de Kuznets ambiental (CKA) y su impacto sobre las negociaciones internacionales. Centro Internacional de Política Económica de la Universidad Nacional de Costa Rica

GRILICHES, Z. (1992): 'The Search for R\&D Spillovers', Scandinavian Journal of Economics, 94, pp. 29-47.

GROSSMAN, G.; y KRUEGER, E. (1991): "Environmental Impacts of a North American Free Trade Agreement," NBER Working Paper, n. 3914.

GROSSMAN, G.; y KRUEGER, E. (1995): "Economic growth and the environment", Quarterly Journal of Economics, v.110, n. 2, pp. 353-377.

HAMILTON, C.; y TURTON, H. (2002): "Determinants of emissions growth in OECD countries", Energy Policy, n. 30, pp. 63-71.

HANG, L.; y TU, M. (2007): "The impacts of energy prices on energy intensity: Evidence from China", Energy Policy, n. 35, pp. 2.978-2.988.

IDAE (2012): Observatorio Energías Renovables, Madrid

IEA (2009): Energy Policies of IEA Countries. Spain 2009 Review, OECD, International Energy Agency, Paris Cedex.

IEA (2009): World Energy Statistics and Balances y Energy Technology R\&D Budgets, International Energy Agency, Paris Cedex.

IEA (2012): IEA Energy Technology R\&D Statistics, Database, International Energy Agency, Paris Cedex.

IM, K. S.; PESARAN, M. H.; y SHIN, Y. (2003): "Testing for unit roots in heterogeneous panels", Journal of Econometrics, n. 115, pp. 53-74.

KAYA, Y. (1989): "Impact of Carbon Dioxide Emission Control on GNP Growth: Interpretation of Proposed Scenarios," paper presented to the Energy and Industry Subgroup, Response Strategies Working Group, Intergovernmental Panel on Climate Change, París, Francia

KREMERS, J. J. M.; ERICSSON, N. R.; y DOLADO, J. J. (1992): "The power of cointegration tests", International Finance Discussion Papers, n. 431, Board of Governors of the Federal Reserve System (U.S.).

LINARES, P. (2002): "Externalidades de la energía y su valoración", en GAGO, A.; y LABANDEIRA, X. (eds.): Energía, Fiscalidad y Medio Ambiente en España; Colección de Estudios de Hacienda Pública, Instituto de Estudios Fiscales, Madrid. 
MAGNANI, E. (2000): "The Environmental Kuznets Curve, environmental protection policy and income distribution", Ecological Economics, Elsevier, vol. 32(3), pages 431-443, March.

MANSFIELD, E.; y SWITZER, L. (1984): "Effects of federal support on company-financed R\&D: the Case of Energy", Management Science, n. 30, pp. 562-571.

MEADOWS, D.H.; MEADOWS, D.L.; RANDERS, J.; y BEHRENS, W. (1972): Los límites del crecimiento, Fondo de Cultura Económica, Madrid

MENDILUCE, M.; PÉREZ-ARRIAGA, I.; y OCAÑA, C. (2010): "Comparison of the evolution of energy intensity in Spain and in the EU15. Why is Spain different?, Energy Policy, n. 38, pp. 639-645.

MMA (2011): Informe de Inventario de emisiones de gases de efecto invernadero de España e información adicional años 1990-2009, Ministerio de Medio Ambiente, y Medio rural y Marino, Madrid

PANAYOTOU, T. (1993):“"Empirical test and policy analysis of environmental degradation at different stages of economic development"; Working Paper, n. 238, Technology and Environment Programme, International Labour Office, Geneva, 1993

PANAYOTOU, T. (1993): "Empirical test and policy analysis of environmental degradation at different stages of economic development"; Working Paper, n. 238, Technology and Environment Programme, International Labour Office, Geneva, 1993

PANAYOTOU, T. (1997): "Demystifying the environmental Kuznets curve: turning a black box into a policy tool",

Environment and Development Economics, n. 2, pp. 465-484.

PEZZEY, J. (1989): "Economic Analysis of Sustainable Growth and Sustainable Development". Environmental department Working Paper, n. 15 (World Bank)

PORTER, M.; y VAN DER LINDE, C. (1995): "Towards a new conception of environment-competitiveness relationship", Journal of Economic Perspectives, n. 9, pp. 97-118

RAVALLION, M.; HEIL, M.; y JALAN, J. (2000): "Carbon emissions and income inequality", Oxford Economic Papers, n. 52: 651-669.

REE (2012): Informe anual 2011, Red Eléctrica Española, Madrid.

ROTHBARD, M. N. (1970): Man, Economy and State. A Treatise on Economic Principles, Los Angeles, CA: Nash Publishing (1962).

RUIZ, M. (2010): "Análisis del impacto de la I+D pública sobre la intensidad energética en la UE-15", Clm. Economía, n. 16, pp. 381-399

SARAVIA, A. (2002): La curva medio ambiental de Kuznets para América Latina y el Caribe, Documento de reflexión académica, n. 23, pp. 10-25, Universidad Mayor de San Simón, Bolivia. 
SCRUGGS, A. (1998): "Political and economic inequality and the environment," Ecological Economics, Elsevier, vol. 26(3), pp. 259-275.

SELDEN, T.; y SONG, D. (1994): "Environmental Quality and Development: Is There a Kuznets Curve for Air Pollution Emissions?", Journal of Environmental Economics and Management, n. 27, v. 2, pp. 147-162.

SHAFIK, N.; y BANDYOPADHYAY, N. (1992): "Economic Growth and Environmental Quality: Time-series and Cross-Country Evidence", World Bank Working Papers, n. 904, pp. 1-6. Washington D.C..

STEGER, U. (1996): "Organization and human resource management for environmental management", GROENEWEGEN, P.; FISCHER, K.; JENKINS, E.G.; y SCHOT, J. (eds.), The Greening of Industry Resource Guide and Bibliography, Washington, D.C.

STOKEY, N. (1998): "Are the limits to growth?", International Economic Review, n. 39, pp. 1-31.

TORRAS, M.; y BOYCE, J. (1998): "Income, inequality, and pollution: a reassessment of the Environmental Kuznets Curve", Ecological Economics, n. 25, pp. 147-160.

UNESA (2010): Memoria 2010, Asociación Española de la Industria Eléctrica UNESA, Madrid

UNESA (2011): Informe estadístico 2011, Asociación Española de la Industria Eléctrica UNESA, Madrid

WCED (1987): Our Common Future, World Commission on Environment and Development, Oxford University Press, Oxford.

WORLD BANK (1992): Development and the environmental; Oxford University Press, 1992),

YUXIANG, K.; y CHEN, Z. (2010): "Government expenditure and energy intensity in China", Energy Policy, n. 38, pp. 691-694. 


\section{APÉNDICE}

\section{ALGUNAS CUESTIONES SOBRE EL MODELO DE LA CURVA AMBIENTAL DE KUZNETS}

El ucraniano emigrado a Estados Unidos, Simón Kuznets (1901-1985), fue Premio Nobel de Economía en 1971 por sus aportaciones a la teoría del crecimiento y la distribución de la renta. Sin embargo, posteriormente, ha sido más conocido por la traslación que se hizo de sus postulados hacia el ámbito de la economía medioambiental, sobre todo, a partir de los trabajos realizados por el Banco Mundial (1992), Shafik y Bandyopadhyay (1992), Panayotou (1993), Selden y Song, 1994), o el de Grossman y Krueger (1995).

Aunque la hipótesis de la CKA no ha estado exenta de críticas (véase De Bruyn, 2000), Magnani (2000) sostiene que existe una más que suficiente evidencia empírica que relaciona el nivel de renta con las emisiones contaminantes $\mathrm{y}$, por tanto, con la calidad ambiental, a pesar de que también hay autores que evitan hablar de calidad ambiental, en la medida de que alude a un stock, mientras que los estudios empíricos utilizan variables flujo (Correa, 2004), y se eluden efectos medioambientales irreversibles producidos en el pasado (Arrow et al., 1995).

En definitiva, la hipótesis de la Curva Ambiental de Kuznets (CKA) analiza la relación existente entre el crecimiento económico y el deterioro medioambiental, tratando de confirmar que, en el corto plazo, el crecimiento económico provoca un mayor deterioro medio ambiental, pero que a largo plazo (en la medida que las economías se hacen más ricas) el crecimiento económico provoca una reducción de la contaminación (una explicación del modelo estructural puede verse en Correa, 2004). Indudablemente, la relación que subyace entre los niveles de contaminación y el crecimiento económico ha sido objeto de diversas interpretaciones que forman parte del modelo básico (estructural), pero que son omitidas en el modelo reducido, que es utilizado en los contrastes empíricos. De hecho, a las críticas de algunos autores en los años noventa en el sentido de que no es posible que el crecimiento económico, por sí mismo, explique el deterioro ambiental, Arrow et al., (1995) responden que, en efecto, el crecimiento no puede ser un sustituto de la política ambiental, considerando que las medidas ambientales tienen un papel relevante y que puede ser recogido dentro del modelo CKA. De la misma manera que, abundando sobre los comportamientos básicos del modelo, Torras y Boyce (1998) introducen en la relación contaminación-crecimiento, la teoría del poder en el ámbito de decisión de las políticas públicas. También, los aportes de Scruggs (1998), y Ravallion et al., (2000), abundan sobre la posibilidad de que la influencia de la desigualdad sobre la contaminación no sea necesariamente en la dirección que afirman Torras 
y Boyce, pudiendo darse un "trade-off" entre emisiones y renta, o bien una relación positiva entre ambas, bajo el supuesto de que la propensión marginal a emitir aumente con la renta.

Otras derivadas del modelo CKA han ido dirigidas a estudiar la relación entre la liberalización del comercio internacional y de los movimientos de factores (globalización) y los niveles de contaminación, como la que lleva a cabo Gitli (2002) al afirmar que hay un efecto composición que impulsa el mix productivo en los países más avanzados hacia actividades menos contaminantes, trasladando las más contaminantes a países menos desarrollados en los que, a su vez, se verían "beneficiados" por el efecto desplazamiento de las industrias más contaminantes, aumentando sus exportaciones de bienes manufacturados. Finalmente, hay otras aportaciones que ponen énfasis en la importancia de la tecnología y de las políticas de impulso, sobre la contaminación (Andreoni y Levinson, 1998), así como otras que consideran al medioambiente como un bien de lujo (Baldwin, 1995; Pezzey, John 1989).

Para terminar, de acuerdo con Correa (2007), en el modelo CKA es posible obtener el punto de quiebra (o punto umbral) donde la renta per cápita alcanza un nivel tal que permite que disminuyan las emisiones contaminantes. El punto de quiebra $\left(\mathrm{Y}^{*}\right)$ se obtiene en la expresión 1 del texto principal, mediante la operación $\mathrm{Y}^{*}=-\beta_{1} / 2 \beta_{2}$, y representa el nivel de renta necesario a partir del cual la sociedad comienza a experimentar reducciones en el nivel de contaminación. Cuanto menor sea el requerimiento de renta, más "efectivas" serán las medidas adoptadas, puesto que menor será el esfuerzo económico a realizar por parte de una sociedad para poder alcanzar un crecimiento económico sostenible.

Son varios los problemas a que se han enfrentado los trabajos empíricos realizados para validar la hipótesis de la CKA. Sin ánimo de exhaustividad, podemos decir que hay tres importantes bloques de aspectos sujetos a controversia en este punto. En primer lugar, los que afectan a las series de datos utilizados, sobre todo, por lo que se refiere a la selección de los contaminantes: en unos casos, la CKA se cumple para contaminantes de efecto local y en otros casos ocurre justo al contrario. Otro tipo de cuestiones controvertidas son las relativas al uso de la técnica de panel para analizar conjuntamente las situaciones de varios países y que suele presentar problemas derivados de una muy diferente estructura de relaciones entre el ingreso y la contaminación entre los distintos países. Finalmente, la especificación concreta de la ecuación reducida que debe someterse a contraste, a veces plantea problemas de interpretación económica. En particular, es sabido que en un compromiso entre la máxima fidelidad al comportamiento real de las variables observadas y la necesidad de obtener evidencia clara sobre el cumplimiento de la relación fundamental que buscamos, el denominado modelo cúbico (especificando una variable adicional PIB3 ${ }_{\mathrm{PC}}$ ) 
constituye el estándar a seguir, puesto que el punto de quiebra suele deparar algo más que el signo de la pendiente de la función estimada; esto es, la propensión marginal a emitir contaminantes no se comporta de manera simétrica a ambos lados de dicho punto, supuesto más convincente que su contrario.

A pesar de ello, la forma de ' $\mathrm{N}$ ' invertida que arrojan muchos de los modelos CKA cúbicos estimados, o bien suele carecer de una explicación económica convincente para el primer tramo descendente de la relación contaminacióningreso, o bien la función estimada no difiere significativamente (para el rango relevante de datos observados) de la función estimada mediante el modelo cuadrático que, al final, es el que se encuentra más generalizado en los trabajos empíricos. En este trabajo no incluimos estimaciones de la variante cúbica del modelo, al resultar en unos peores ajustes que los obtenidos con el modelo cuadrático. 